OPEN ACCESS

Edited by: Matthias Altmeyer, University of Zurich, Switzerland

Reviewed by: Judith Mine-Hattab, Institut Curie, France Gary Karpen, University of California, Berkeley, United States

*Correspondence: Philipp Oberdoerffer PO@jhmi.edu

${ }^{\dagger}$ Present address: Philipp Oberdoerffer, Department of Radiation Oncolgy and Molecular Radiation Sciences, Johns Hopkins University School of Medicine, Baltimore, MD, United States

Specialty section:

This article was submitted to Human and Medical Genomics, a section of the journal Frontiers in Genetics

Received: 23 July 2021 Accepted: 08 October 2021 Published: 21 October 2021

Citation:

Sebastian R, Aladjem MI and Oberdoerffer $P$ (2021) Encounters in

Three Dimensions: How Nuclear Topology Shapes Genome Integrity.

Front. Genet. 12:746380. doi: 10.3389/fgene.2021.746380

\section{Encounters in Three Dimensions: How Nuclear Topology Shapes Genome Integrity}

\author{
Robin Sebastian ${ }^{1}$, Mirit I. Aladjem ${ }^{1}$ and Philipp Oberdoerffer ${ }^{2 * t}$ \\ ${ }^{1}$ Developmental Therapeutics Branch, Center for Cancer Research, National Cancer Institute, NIH, Bethesda, MD, United States, \\ ${ }^{2}$ Division of Cancer Biology, National Cancer Institute, NIH, Rockville, MD, United States
}

Almost 25 years ago, the phosphorylation of a chromatin component, histone H2AX, was discovered as an integral part of the DNA damage response in eukaryotes. Much has been learned since then about the control of DNA repair in the context of chromatin. Recent technical and computational advances in imaging, biophysics and deep sequencing have led to unprecedented insight into nuclear organization, highlighting the impact of threedimensional (3D) chromatin structure and nuclear topology on DNA repair. In this review, we will describe how DNA repair processes have adjusted to and in many cases adopted these organizational features to ensure accurate lesion repair. We focus on new findings that highlight the importance of chromatin context, topologically associated domains, phase separation and DNA break mobility for the establishment of repair-conducive nuclear environments. Finally, we address the consequences of aberrant 3D genome maintenance for genome instability and disease.

Keywords: genome integrity, nuclear organization, replication stress, Topologically Associated Domain, chromatin, DNA double-strand break repair, phase separation

\section{INTRODUCTION}

Eukaryotic genomes are exposed to numerous sources of DNA damage, of which DNA doublestrand breaks (DSBs) are arguably the most deleterious. DSBs can arise from exposure to genotoxic agents, many of which are used in cancer therapy, but they can also be the result of endogenous processes such as oxidative metabolism and DNA replication. Aberrant repair of DSBs can cause chromosomal translocations, genomic duplications or deletions, as well as DNA mutations, all of which may result in defective cell function, cell death or malignant transformation.

Three main pathways exist to repair DSBs in mammalian cells: i) non-homologous end-joining (NHEJ), a fast but error-prone re-ligation of broken DNA ends; ii) microhomology-mediated end joining (MMEJ, also known as alternative end joining or alt-EJ), a process that relies on moderate DNA end resection and frequently results in small insertions or deletions (indels); and iii) homologous recombination (HR), which is a templated process and therefore considered errorfree, but generally restricted to S/G2 phases of the cell cycle (Jasin and Rothstein, 2013; Chang et al., 2017). While both error-free and potentially error-prone repair pathways play essential roles in genome maintenance, inappropriate repair pathway choice can have detrimental consequences for genome integrity (Scully et al., 2019). The latter can be the result of DNA repair factor mutations, as often observed in cancer cells (e.g., breast and ovarian cancers with defective BRCA genes), but further depends on a more complex set of temporal and local factors, most notably cell cycle phase and the DSB-surrounding nuclear environment. Defects in a given repair pathway or inappropriate repair pathway choice can be exploited for synthetic lethal cancer therapy approaches, such as 
poly(ADP-ribose) polymerase 1 (PARP1) inhibition, which selectively kills HR-deficient cancers (Lord and Ashworth, 2017).

Recent advances in biochemical, biophysical, imaging and deep sequencing technologies have led to unprecedented insight into nuclear organization and its changes with time and/or in response to cell-intrinsic or -extrinsic perturbations (Rowley and Corces, 2018; Kempfer and Pombo, 2020). Not surprisingly, there is an intimate link between genome organization, DNA accessibility and the functional regulation of DNA transactions, such as transcription, replication and DNA repair (Dekker and Mirny, 2016; Misteli, 2020). A first and by now well-characterized barrier to DNA access is the chromatin fiber, in which DNA is wrapped around positively charged nucleosomes consisting of a histone octamer, which typically comprises two copies of the core histones $\mathrm{H} 2 \mathrm{~A}, \mathrm{H} 2 \mathrm{~B}, \mathrm{H} 3$ and H4. Depending on the cellular context, core histones can be replaced with specialized histone variants. The impact of nucleosome composition and remodeling as well as static and dynamic histone modifications on DSB repair has been extensively investigated and we refer the reader to several excellent reviews summarizing this work (Price and D’Andrea, 2013; Lebeaupin et al., 2015; Ferrand et al., 2021; Hauer and Gasser, 2017). How the three-dimensional (3D) organization of the chromatin fiber in nuclear space can affect DSB repair, and conversely, be affected by the latter, is significantly less well understood.

Using a combination of high-throughput sequencing-based conformation capture approaches and fluorescent in situ hybridization (FISH)-based imaging, higher order chromatin organization can be interrogated at the kilobase $(\mathrm{kb})$ or $\mathrm{nm}$ scale, revealing complex chromatin looping that is often tied to cell cycle phases or DNA transactions such as transcription or replication (Bickmore, 2013; Rao et al., 2014; Rowley and Corces, 2018; Misteli, 2020). Chromatin loops are both architectural and functional in nature, providing a platform for $3 \mathrm{D}$ genome compaction as well as regulatory interactions. The size of chromatin loops can range from tens of $\mathrm{kb}$ to several $100 \mathrm{~kb}$, often containing loops within loops. Loops that are characterized by unique chromatin features are referred to as topologically associated domains (TADs). Recent advances have provided significant insight into the processes that promote and maintain TAD formation, which involves active loop extrusion supported by architectural proteins such as CTCF and the structural maintenance of chromosomes (SMC) cohesin complex (Fudenberg et al., 2017; Rao et al., 2017; Davidson and Peters, 2021). Single cell analyses demonstrate that TAD formation is highly dynamic and often only detectable in a small subset of cells at any given time (Finn et al., 2019). For detailed reviews of recent advances in our understanding of TAD formation and function we refer the reader to (Hansen et al., 2018; Szabo et al., 2019). Of note, individual TADs can segregate into larger chromatin domains, which differ in loop density as well as nucleosome composition and mobility (Janssen et al., 2018; Szabo et al., 2019). While the formation of such chromatin domains is associated with TAD-specific histone modifications, recent findings demonstrate that liquid phase separation may help organize the heterochromatin compartment and perhaps
TAD organization more generally (Larson and Narlikar, 2018; Gibson et al., 2019; Sanulli et al., 2019).

In this review, we will describe recent insight into the orchestration of DSB repair in the context of nuclear topology. Specifically, we will discuss how DSB repair processes have adjusted to and in many cases adopted the organizing principles of the nucleus to ensure accurate lesion repair. Finally, we will briefly address the consequences of failed 3D genome maintenance for genome instability and disease.

\section{LOCATION, LOCATION LOCATION - CHROMATIN CONTEXT AFFECTS REPAIR OUTCOME}

Large-scale interdisciplinary efforts such as the Encyclopedia of DNA elements (ENCODE) or the 4D Nucleome Projects have helped to map the composition of mammalian chromatin with remarkable resolution (Consortium, 2004; Dekker et al., 2017; Consortium et al., 2020). As we continue to obtain more refined insight into how DNA is organized into functionally and phenotypically distinct chromatin domains, it is time to revisit how these domains affect genome integrity.

\section{Impact of Chromatin Context on DNA Double-Strand Break Repair Pathway Choice}

Our understanding of the many chromatin modifications that interface with DSB repair processes is growing continuously and has been extensively reviewed elsewhere (Dabin et al., 2016; Hauer and Gasser, 2017; Ferrand et al., 2021). Nevertheless, a systematic assessment of DSB repair outcome across the various distinct chromatin states that coexist in a single cell has been missing to date. The advent of CRISPR/Cas9 as an effective means to target DSB induction to any given genomic location provides an opportunity to address this knowledge gap. Recent work by van Steensel and colleagues pioneered this effort by combining a multiplexed genome editing approach with a reporter that can distinguish between the two major errorprone DSB repair pathways, MMEJ and NHEJ (Schep et al., 2021). By overlaying a highly quantitative, sequencing-based "DNA repair scar"-counting readout with existing epigenome data, comprehensive MMEJ and NHEJ repair maps were generated for chromatin contexts across $>1,000$ genomic locations. This work complements and extends previous genome-wide assessments of HR versus NHEJ usage using the AsiSI endonuclease, which cuts the human genome efficiently at $<100$ sites and revealed a preference for HR in transcribed genomic regions (Aymard et al., 2014). Notably, MMEJ, which like HR relies on the resection of broken DNA ends to expose patches of homology for break alignment and repair, was found to be more frequent in specialized heterochromatic chromatin environments marked by $\mathrm{H} 3$ trimethylated at K27 (H3K27me3) (Schep et al., 2021). Together, these findings suggest that despite a common initial end processing step, HR and MMEJ are differentially 
controlled by chromatin context, perhaps by regulating the shift from short-range resection to long-range resection generally associated with HR (Symington and Gautier, 2011; Scully et al., 2019).

Supporting a functional role for $\mathrm{H} 3 \mathrm{~K} 27 \mathrm{me} 3$ in modulating DSB repair outcome, inactivation of $\mathrm{EZH} 2$, the histone methyltransferase responsible for most of its deposition, caused a shift in DNA repair away from MMEJ towards NHEJ (Schep et al., 2021). Moreover, EZH2 inhibition was recently shown to shift repair from HR to NHEJ in some ovarian cancer cell lines (Karakashev et al., 2020). Rather than being a lesionspecific effect of $\mathrm{H} 3 \mathrm{~K} 27 \mathrm{me} 3$, the reduction in HR efficiency upon EZH2 inhibition was due to transcriptional de-repression of MAD2L2, a component of the Shieldin complex that counteracts DNA end resection (Karakashev et al., 2020). Consistent with the defect in HR, EZH2 inhibition selectively sensitized ovarian cancer cell lines with sufficiently high MAD2L2 levels to PARP inhibitors in both orthotopic and patient-derived xenografts. Seemingly in contrast to these findings, EZH2 inhibition has recently been associated with replication fork stabilization and PARPi resistance in BRCA2deficient and hence HR-defective breast cancer cells (Rondinelli et al., 2017). EZH2-mediated destabilization of replication forks involved H3K27me3 mediated recruitment of the MUS81 endonuclease, thus coupling histone modification to replication fork protection. Together, these observations emphasize that a widely distributed mark of facultative heterochromatin such as $\mathrm{H} 3 \mathrm{~K} 27 \mathrm{me} 3$ can have a complex impact on genome maintenance, which likely depends on genomic context as well as the type of DNA lesion. Consequently, manipulation of EZH2 resulted in cell linespecific, yet predictable outcomes in response to genotoxic therapy.

The macro-histone variant macroH2A1, which frequently colocalizes with $\mathrm{H} 3 \mathrm{~K} 27 \mathrm{me} 3$ domains across the genome (Chen et al., 2014), has recently emerged as another modulator of DNA repair pathway choice (Ruiz et al., 2019; Sebastian et al., 2020). Specifically, macroH2A1 controls DSB repair via balanced expression of its two alternative splice variants, macroH2A1.1 and macroH2A1.2. MacroH2A1.1, which unlike macroH2A1.2 can bind poly (ADP-ribose) (PAR), interacts with the MMEJ effectors PARP1 and Ligase 3 in a PAR-dependent manner to facilitate MMEJ, whereas macroH2A1.2 promotes $\mathrm{HR}$ by facilitating BRCA1 recruitment to sites of DNA damage (Khurana et al., 2014; Kim et al., 2018). Deletion of macroH2A1.2 shifts repair towards MMEJ resulting in genome instability that is particularly pronounced at the macroH2A1-and $\mathrm{H} 3 \mathrm{~K} 27 \mathrm{me} 3$-rich inactive $\mathrm{X}$ chromosome in female mouse fibroblasts (Sebastian et al., 2020). If and how macroH2A1 and EZH2 functions are related during DSB repair and/or replication stress remains to be determined. However, deregulation of $\mathrm{H} 3 \mathrm{~K} 27 \mathrm{me} 3$ levels or macroH2A1 variant expression, and the associated HR defects, have both been linked to PARP inhibitor resistance, chromosomal abnormalities, and [...] PARP inhibitor resistance and chromosomal instability in cancer cells in cancer cells (Khurana et al., 2014; Karakashev et al., 2020).
Together, these recent advances exemplify the impact of improved integrative analyses of chromatin composition on our understanding of genome maintenance. They further emphasize the need to i) consider functionally distinct proteoforms, often as the result of alternative splicing, and ii) distinguish lesion-specific from global effects of chromatin perturbation such as the epigenetic deregulation of repair factors.

\section{Chromatin Domains Guide DNA Replication}

Recent work suggests that, much like distinct chromatin domains differentially affect DSB repair factor recruitment, they can modulate the initiation and progression of DNA replication, as well as the repair of stalled replication forks (Alabert et al., 2017; Aladjem and Redon, 2017; Bellush and Whitehouse, 2017). While the impact of chromatin on replication timing and DNA polymerase processivity is well described (Marchal et al., 2019; Klein et al., 2021), it was perhaps unexpected that chromatin composition can determine the choice of DNA replisome subunits. Comparative analysis of two replisome-associated proteins involved in the cellular response to replication stress, the translocase FANCM and the poorly characterized DONSON protein (Reynolds et al., 2017), uncovered the existence of distinct replisome complexes. While both proteins facilitate the repair of DNA interstrand crosslinks (ICLs) via a lesion traverse mechanism, FANCM-associated replisomes are most prevalent in late stages of S phase, generally induced by replication stress, and colocalize with a chromatin environment characteristic of late replicating and fragile DNA (Zhang et al., 2020). DONSON, on the other hand, appears to form a distinct replisome complex that is primarily responsible for ICL traverse in early $\mathrm{S}$ phase. Notably, FANCM and DONSON show the same bias in replication timing- and chromatin domain-association in cells without ICLs. How distinct chromatin environments regulate replisome composition remains to be determined, as does the functional relevance and potential clinical implications of having different replisomes act throughout $S$ phase. Of note, defects in DONSON or FANCM manifest in microcephalic dwarfism and breast cancer susceptibility, respectively (Reynolds et al., 2017; Catucci et al., 2018). It will be interesting to investigate whether or not these distinct pathological outcomes relate to the observed differences in replication stress responses.

Altogether, we anticipate that continued, refined and comprehensive mapping of functionally distinct chromatin components, DNA repair outcome and genetic dependencies will provide a wealth of clinically actionable insight into repair mechanisms.

\section{DNA DOUBLE-STRAND BREAK REPAIR DOMAINS - NEW INSIGHTS INTO FORMATION AND FUNCTION}

Beyond its role in modulating and regulating DNA transactions, chromatin shapes and defines the formation of functionally distinct, specialized nuclear environments. Recently, it has become apparent that DNA repair takes advantage of these features to form contained and often pathway-specific micro- 
environments, sequestering DNA lesions for reasons that remain to be fully investigated, but may help prevent illegitimate and potentially harmful repair events. Several novel concepts highlight and extend the impact of nuclear organization to genome maintenance and are discussed below.

\section{Chromatin Loop Extrusion: The DNA Repair Focus Revisited}

Microscopically visible DNA damage response (DDR) foci are a striking feature of DSB repair (Rogakou et al., 1999). These foci generally reflect a single DNA lesion and its association with a plethora of often repair pathway-specific damage sensors and repair factors that can cover several hundred kilobases $(\mathrm{kb})$ of lesion-surrounding DNA. At the heart of most DSB-associated chromatin changes is the phosphorylation of S139 on the histone $\mathrm{H} 2 \mathrm{~A}$ variant $\mathrm{H} 2 \mathrm{AX}$ (referred to as $\gamma \mathrm{H} 2 \mathrm{AX}$ ), orchestrated by early DNA damage signaling events involving one or more of three PI3K family kinases - ATM, ATR and DNA-PKcs (Rogakou et al., 1998; Smeenk and van Attikum, 2013). $\gamma \mathrm{H} 2 \mathrm{AX}$ facilitates the recruitment of key downstream repair effector proteins via the $\gamma \mathrm{H} 2 \mathrm{AX}$-binding MDC1 scaffold protein (Smeenk and van Attikum, 2013). Although DSB-surrounding $\gamma \mathrm{H} 2 \mathrm{AX}$ chromatin domains have been mapped across the genome in response to numerous DNA damaging agents or endonucleases, the molecular basis that underlies the formation of up to megabase $(\mathrm{Mb})$ size regions of $\gamma \mathrm{H} 2 \mathrm{AX}$ has long puzzled the field (Iacovoni et al., 2010). Of note, $\gamma \mathrm{H} 2 \mathrm{AX}$ domain boundaries were found to coincide with topologically associated domain (TAD) boundaries (Caron et al., 2012), and super-resolution light microscopy revealed that CTCF, a TAD boundary marker, is juxtaposed to $\gamma \mathrm{H} 2 \mathrm{AX}$ foci (Natale et al., 2017). Similar observations were reported for the 53BP1 repair factor, the recruitment of which depends on the RNF8/RNF168 E3 ubiquitin ligases, which in turn bind MDC1 (Hustedt and Durocher, 2016; Ochs et al., 2019). Together, these observations suggest that DNA repair domain formation is governed by high-order chromatin organization.

The organization of the genome into TADs involves ATPdependent, active extrusion of DNA loops through a cohesin ring (Rao et al., 2017; Ganji et al., 2018; Davidson et al., 2019; Davidson and Peters, 2021). Cohesin consists of the SMC1SMC3 heterodimeric adenosine triphosphatase (ATPase), the SMC protein partner RAD21 and either of the helical repeat proteins STAG1 or STAG2, and was originally identified as a mediator of sister chromatid cohesion. TAD loops are anchored by inverted CTCF sites, which terminate cohesion-mediated loop extrusion when encountered on opposing strands through yet to be determined mechanisms. For a detailed overview of cohesin function in TAD formation, we refer the reader to a number of excellent reviews (Fudenberg et al., 2017; Rowley and Corces, 2018; Yatskevich et al., 2019).

Loop extrusion does not only facilitate the organization of TADs and chromatin domains, it can also facilitate the ligation of otherwise distal DNA ends. This was first described for the processes of VDJ and class switch recombination, which mediate the rearrangement and assembly of immunoglobulin
(Ig) gene elements that are up to several $100 \mathrm{~kb}$ apart (Zhang et al., 2019a; Zhang et al., 2019b). Like TAD formation, VDJ recombination and class switching depend on CTCF-associated cohesin rings to allow for accurate ligation of matching gene elements (Ba et al., 2020; Dai et al., 2021). Notably, cohesin was found to accumulate at DSB sites other than the Ig locus, and its depletion resulted in genome instability (Ström et al., 2004; Ünal et al., 2004; Potts et al., 2006; Covo et al., 2010; Meisenberg et al., 2019). Recruitment of cohesin to DSBs was observed throughout the cell cycle, suggesting a function independent of sister chromatin adhesion or HR (Ström et al., 2004; Potts et al., 2006; Caron et al., 2012; Meisenberg et al., 2019). Together with the finding that $\gamma \mathrm{H} 2 \mathrm{AX}$ domains overlap with TADs (Caron et al., 2012; Natale et al., 2017), these observations point to a role for loop extrusion in the formation of DSB repair domains. Experimental support for the latter came recently from an elegant set of analyses combining chromosome conformation capture mapping, chromatin immunoprecipitation (ChIP) and AsiSI-mediated DSB induction (Arnould et al., 2021). A marked discrepancy was observed between the distribution of $\gamma \mathrm{H} 2 \mathrm{AX}$ and ATM, the kinase primarily responsible for DSB-induced $\mathrm{H} 2 \mathrm{AX}$ phosphorylation (Arnould et al., 2021). In contrast to the TAD-sized $\gamma \mathrm{H} 2 \mathrm{AX}$ domains, ATM accumulation was restricted to the immediate vicinity of the DSB, suggesting that $\mathrm{H} 2 \mathrm{AX}$ phosphorylation is not mediated by the linear spreading of the kinase across TADs. Using tightly controlled DSB synchronization and release combined with ATM inhibition and/or deletion of several cohesion subunits, the authors provide compelling evidence for a model in which $\mathrm{H} 2 \mathrm{AX}$ containing nucleosomes are rapidly phosphorylated as they actively pass by DSB-anchored cohesin. TADs thus delineate the boundaries of $\gamma \mathrm{H} 2 \mathrm{AX}$ chromatin domains in a manner that involves one-sided loop extrusion on either side of the break. Importantly, this process was conserved in yeast (Arnould et al., 2021), and the observed kinetics are consistent with previously reported rates of $\gamma \mathrm{H} 2 \mathrm{AX}$ foci assembly (Ochs et al., 2019). Moreover, DNA damage was shown to result in the ATMdependent strengthening of existing TAD structures, perhaps as a mechanism to protect $3 \mathrm{D}$ genome integrity during DNA repair (Sanders et al., 2020). Together, these findings highlight how chromosome conformation and TAD-associated loop extrusion have been adopted by the DDR to ensure repair domain formation and genome maintenance (Figure 1).

\section{Phase Separation of Double-Strand Break Repair Domains?}

In addition to structured chromatin organization, nuclear subdomains can be organized by physicochemical forces (Boeynaems et al., 2018). Examples of such domains include PML bodies, Cajal Bodies, nuclear speckles, and the nucleolus, which were proposed to behave as semifluid spheres suspended in semifluid nucleoplasm almost 2 decades ago (Handwerger et al., 2005). Experimental evidence for the physical nature of such assemblies was provided in 2009, when P granules (RNA and protein-containing bodies) were shown to display liquid-like 

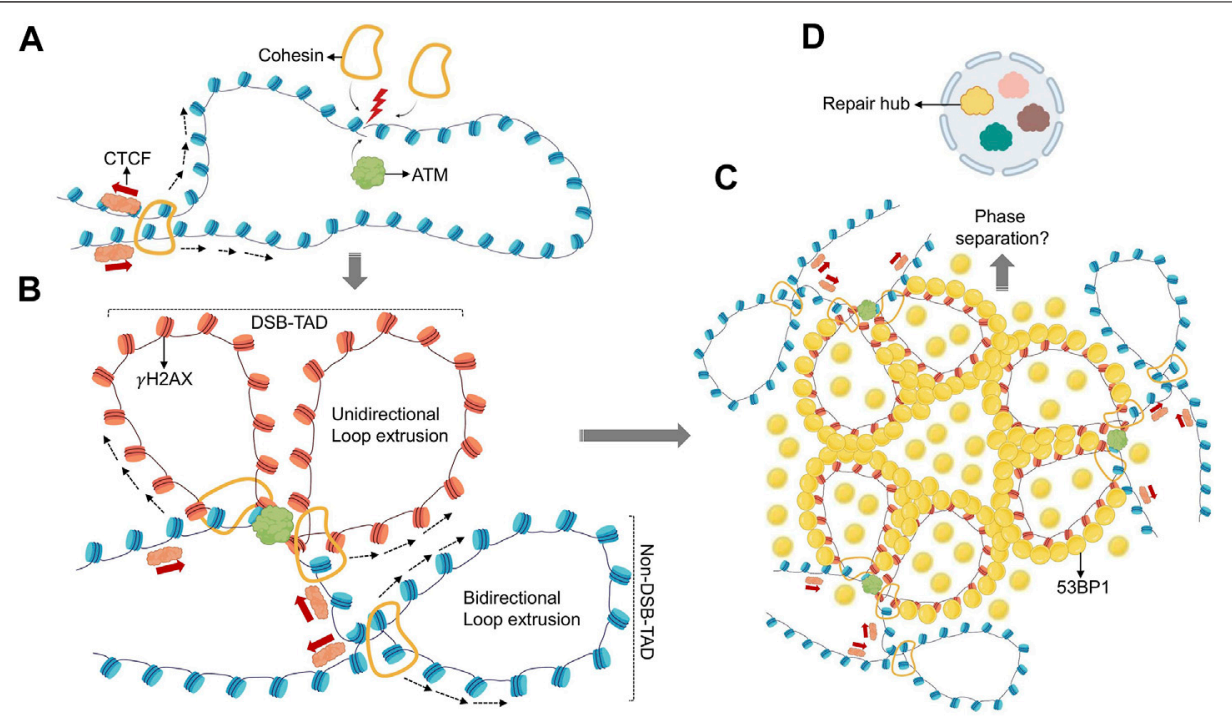

FIGURE 1 | Role of loop extrusion in establishing repair domains. (A) DSBs initiate recruitment of ATM kinase and the cohesin complex. (B) DSB-associated cohesin anchors initiate unidirectional loop extrusion at both DSB ends, towards TAD anchors. ATM phosphorylates H2AX while nucleosomes are extruded ( $\gamma \mathrm{H} 2 \mathrm{AX}$ nucleosomes are shown in red). Loop extrusion stops when existing TAD boundaires are encountered. This process generates a TAD-overlapping $\gamma \mathrm{H} 2 \mathrm{AX}$ domain. (C) $\gamma \mathrm{H} 2 \mathrm{AX}$ domains recruit 53BP1 repair factors creating similar, TAD-overlapping 53BP1 profiles. (D) 53BP1 mediated phase separation at TAD-associated 53BP1 domains may promote higher-order assembly of multiple 53BP1-TADs, and possibly multiple DSBs, to create spatially segregated repair hubs (yellow). Distinct nuclear subcompartments are symbolized in different colors.
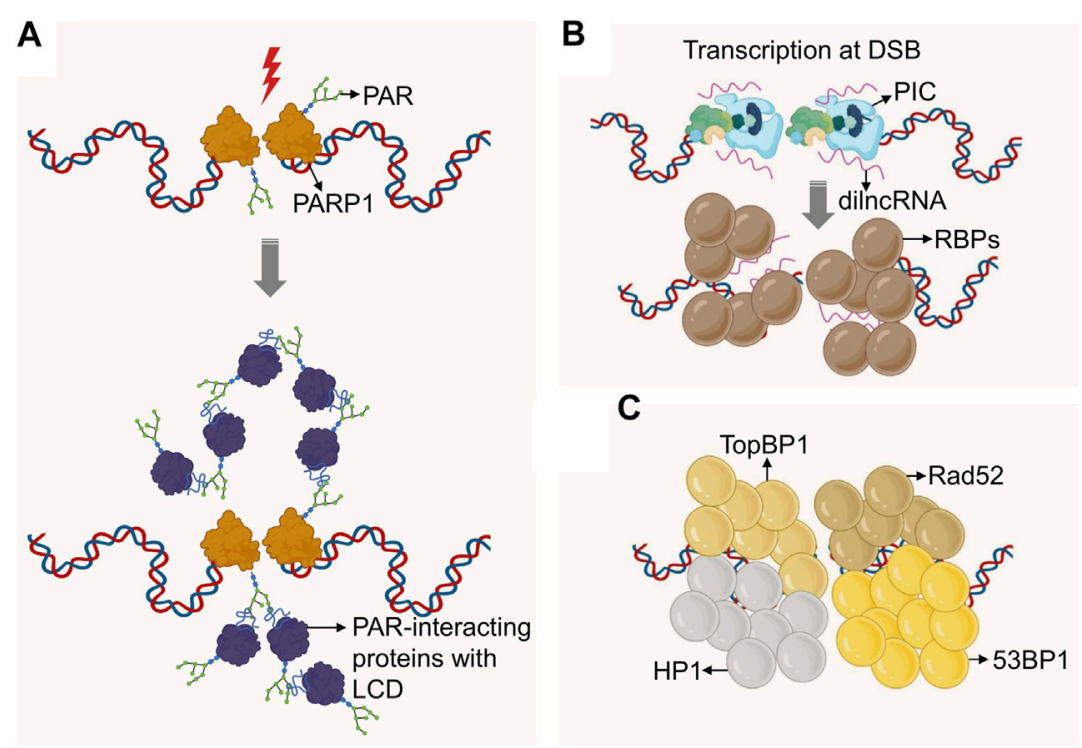

FIGURE 2 | Formation of DNA repair domains via phase separation. (A) DSBs recruit PARP1, which mediates DSB-proximal PARylation, attracting PAR-binding proteins, many of which contain Low Complexity Domains (LCD). The latter promote molecular crowding and concomitant phase separation. (B) DSBs recruit RNA Polymerase II, which initiates transcription at the DSB site to generate dilncRNAs. These RNAs are bound by IDR-containing RNA binding proteins (RBPs) which can drive phase separation. (C) Protein modifications at DSBs recruit proteins such as 53BP1, TopBP1, Rad52 and HP1, all of which were shown to form higher order condensates via phase separation. If these domains are distinct or can be fused remains to be determined. 53BP1 may further promote phase separation via its binding to dilncRNA.

properties and form by phase separation in C. elegans (Brangwynne et al., 2009). By definition, phase separation in biological systems occurs when a homogenous mixture of macromolecules such as proteins or nucleic acids in a solution spontaneously separate into a phases of distinct densities. In the context of chromatin, condensates can form either via bridging of 
nucleosome-binding proteins (polymer-polymer phase separation) or via multivalent interactions among soluble, chromatin-associated proteins (liquid-liquid phase separation) (Erdel and Rippe, 2018; Miné-Hattab and Taddei, 2019). In the case of liquid-liquid phase separation, which is the focus of this section, the dense phase has liquid-like properties, no fixed stochiometry and accumulates certain macromolecules. Since the non-dense phase is depleted of said macromolecules, it allows the dense phase to attain a compartment-like status. Phase-separated liquid condensates can eventually form more solid-like states exhibiting different material properties, such as dynamic liquid-like droplets or less dynamic gels and solid amyloids (Banani et al., 2017; Miné-Hattab and Taddei, 2019).

Studies looking at the protein composition of phase-separated biological condensates suggest multivalency of adhesive domains and linear motifs as defining features of proteins that drive phase separation. Prominent examples are intrinsically disordered regions (IDRs) and Low Complexity Domains (LCDs). Of note, recent work from the Narlikar and Karpen labs demonstrated that phase separation is also an organizing principle for chromatin domains, particularly heterochromatin (Larson et al., 2017; Strom et al., 2017; Larson and Narlikar, 2018). It may thus be not surprising that phase separation was found to contribute to the formation of DNA repair microenvironments. Underlying mechanistic insight and possible consequences for genome maintenance are discussed below, separated by the phase-separating properties of primary responders or effectors of the DNA damage response (Figure 2).

\section{Phase Separation by Poly (ADP-Ribose)}

PARP1 is an abundant nuclear protein that attaches a negatively charged (PAR) polymer to itself and to multiple target proteins. This modification is one of the earliest events in the DNA damage response against a wide variety of DNA lesions (Kraus, 2020). Consistent with this, PARylation has been implicated in the repair of single-strand breaks (SSBs), DSBs, the stabilization of DNA replication forks and the modification of the DNA damage-associated chromatin (Ray Chaudhuri and Nussenzweig, 2017). While PARP1 itself has no IDRs, its activation at sites of DNA damage was found to promote transient phase separation via the formation of PAR chains. PAR chains act as a molecular scaffold for the assembly of proteins with disordered or low complexity domains, thereby initiating demixing of distinct liquid phases to achieve dynamic intracellular compartmentalization. Two types of LCDs participate in this process: positively charged arginine-glycine-glycine (RGG) repeats, which act as a PAR sensor, and prion-like protein domains, which amplify PARseeded liquid demixing (Altmeyer et al., 2015). This process appears to reflect a general mechanism to dynamically reorganize the soluble nuclear space in response to DNA lesions (Figure 2A). Recent work has implicated the highly disordered RGG containing Fused in Sarcoma (FUS/TLS) protein in PARseeded liquid demixing (Singatulina et al., 2019). FUS, together with EWS and TAF15, is a member of the FET family and one of the most abundant and highly PARylated nuclear RNA-binding proteins (Britton et al., 2014; Singh et al., 2015; Zhen et al., 2017). FUS condensates have liquid-like properties, the dynamics and structure of which are affected by pathogenic mutations as well as LCD phosphorylation (Patel et al., 2015; Monahan et al., 2017; Murray et al., 2017). Upon DNA damage, the C-terminal RGG repeats of FUS form repair domains in response to PARP activity in a transient and reversible manner (Singatulina et al., 2019). PAR-seeded liquid demixing may thus facilitate the compartmentalization of damaged DNA, and its functional relevance for the DDR is a subject of intense investigation.

\section{Phase Separation by RNA}

Analogous to PAR chains, nucleic acids were shown to seed phase-separated structures by recruiting IDR-containing RNA binding proteins (RBPs). Non-coding RNAs (ncRNAs) form molecular scaffolds that connect multiple RBPs into a dynamic network of phase separated droplets (Lin et al., 2015; Aumiller et al., 2016; Pessina et al., 2019; Guo et al., 2021). Messenger RNA (mRNA) was also found to form phase-separated droplets. However, in this case the seed involved specific $3 \mathrm{D}$ structures through complementary RNA base pairing (Langdon et al., 2018). Of note, growing evidence points to DNA damage-induced transcription of non-coding RNA at DNA break sites (Sebastian and Oberdoerffer, 2017; Zong et al., 2020). These DNA damage induced long non-coding RNAs (dilncRNA) were found to be necessary for DNA damage response (DDR) focus formation (Francia et al., 2012), form transient RNA:DNA hybrids (Wahba et al., 2013; Ohle et al., 2016), and regulate the extent of end resection and consequently HR. Recently, it was shown that the induction of DSBs resulted in the assembly of functional promoters that include a complete RNA polymerase II preinitiation complex, MED1 and CDK9 (Pessina et al., 2019). Mediator and RNA polymerase II clusters are known to associate in transcription-dependent condensates (Cho et al., 2018), and depletion or inactivation of these factors caused a reduction in DDR foci. Moreover, dilncRNAs drove molecular crowding of DDR proteins, such as 53BP1, into foci that behave like phaseseparated condensates (Pessina et al., 2019). Given that phase separation has been proposed as a mechanism for transcription control (Hnisz et al., 2017), a similar role in the DDR may provide an intriguing rationale for DSB-associated transcripts (Figure 2B).

\section{Phase Separation by DNA Repair Factors}

Phase separation can also be mediated by DNA repair proteins. The NHEJ effector and chromatin binding protein 53BP1 was recently shown to condensate into repair domains that are dynamic and show droplet-like behavior (Figure 2C). Repair domain formation by 53BP1 undergoes frequent fusion and fission events, is highly sensitive to changes in osmotic pressure, temperature, salt concentration and the disruption of hydrophobic interactions, consistent with liquid demixing (Kilic et al., 2019). Light-induced optoDroplet formation experiments (Taslimi et al., 2014; Shin et al., 2017) combined with 53BP1 mutagenesis suggest that a $\mathrm{C}$-terminal multivalent domain as well as the C-terminal BRCT domain are sufficient for 53BP1 phase separation properties. The implication of the BRCT domain is intriguing as other BRCT-containing protein such as BRCA1 did not appear to phase-separate, suggesting sequence specificity and/ or more complex organizing principles. Providing functional 
insight into possible roles of DNA damage-induced phase separation, the tumor suppressor protein p53 was found enriched within 53BP1 repair domains, and conditions that perturb 53BP1 phase separation negatively affected 53BP1dependent activation of p53 (Kilic et al., 2019). However, a direct role for 53BP1-mediated droplet formation in the repair of DSBs has not been identified to date.

53BP1 foci have regulatory functions beyond the immediate repair of DSBs. Cells that carry replication stress-associated DNA damage, such as lesions resulting from under-replicated DNA, into the next cell cycle form so called 53BP1 nuclear bodies (Harrigan et al., 2011; Lukas et al., 2011). Like DSB-induced 53BP1 foci, 53BP1 nuclear bodies were sensitive to osmotic stress, indicative of phase separation properties (Kilic et al., 2019). Nuclear body formation appears to inhibit repair in G1 to facilitate templated, RAD52-mediated repair of the lesion in the next $S$ phase (Lezaja and Altmeyer, 2018; Spies et al., 2019). Notably, RAD52 was shown to form liquid droplets in Saccharomyces cerevisiae (Oshidari et al., 2020). RAD52 droplets cooperate with DNA damage-inducible intranuclear microtubule filaments to promote the clustering of DNA damage sites and facilitate HR (Oshidari et al., 2020). Recent studies suggest that RAD52 may be a "client" rather than a "scaffold" for liquid droplets, pointing to additional factors involved in their formation (Miné-Hattab et al., 2021). It will be interesting to determine if a dynamic transition exists between 53BP1 nuclear bodies and RAD52 droplet formation, which may help regulate repair activity at 53BP1 nuclear bodies in a cell cycle-dependent manner.

A number of other DSB repair-associated proteins have been reported to exhibit phase separation properties (Figure 2C). A notable example is heterochromatin protein 1 (HP1), which can associate with sites of DNA damage to aid the recruitment of 53BP1 and RAD51 (Alagoz et al., 2015). HP1 has been implicated in the evolutionarily conserved, liquid-liquid phase separation of heterochromatin domains (Larson et al., 2017; Strom et al., 2017; Sanulli et al., 2019; Wang et al., 2019). However, both the precise nature of HP1 subcompartment formation and its potential role at DSBs remain to be determined (Chiolo et al., 2011; McSwiggen et al., 2019; Erdel et al., 2020). More recently, the ATR activator TopBP1 was shown to self-assemble into micron-sized condensates. Single amino acid substitutions of key residues in the ATR-activation domain of TopBP1, which also contains IDRs, disrupt TopBP1 condensation and, consequently, ATR/ Chk1 signaling and replication fork stalling (Frattini et al., 2021). Of note, DSB-dependent formation of early DDR events such as $\gamma \mathrm{H} 2 \mathrm{AX}$ and MDC1 foci did not exhibit liquid-like properties (Kilic et al., 2019), pointing to distinct and likely dynamic modes of DSB micro-environment organization. The latter is further consistent with the seemingly independent and/or complementary initiation of phase separation initiation at DNA lesions via either PAR, RNA or DNA repair factors.

\section{Coordination Between Topologically Associated Domains and Phase Separation in DNA Repair}

Recent evidence suggests that the formation of phase-separated repair environments is tightly linked to $\mathrm{TAD}$-associated repair micro-domains. Using super-resolution microscopy, Lukas and colleagues were able to provide unprecedented insight into repair domain formation by 53BP1 (Ochs et al., 2019). Specifically, 53BP1 and its interacting factor RIF1 were found to form an autonomous functional module that stabilizes $3 \mathrm{D}$ chromatin topology at sites of DNA breakage. This process involves the sequential accumulation of 53BP1 at TAD-associated, compact chromatin, followed by RIF1 accumulation at the boundaries between these domains. The alternating distribution of 53BP1 and RIF1 was found to stabilize neighboring TADs into a higher-order arrangement surrounding a single DSB. Depletion of $53 \mathrm{BP} 1$ or RIF1 led to the de-condensation of DSB-surrounding chromatin and aberrant spreading of DNA repair factors. Of possible functional relevance, depletion of either protein also resulted in hyper-resection of DNA ends (Ochs et al., 2019).

Interestingly, topological distortions of 53BP1 domains could also be observed upon depletion of cohesin (Ochs et al., 2019). Together with recent insight into TAD-dependent DDR focus formation, these findings suggest a staged model wherein TAD structure dictates $\gamma \mathrm{H} 2 \mathrm{AX}$ foci formation, which in turn promotes their DNA repair-independent, 53BP1-mediated higher-order assembly, perhaps in a process that involves phase separation (Figure 1). More work is needed to understand the implications of this $3 \mathrm{D}$ re-organization of the DSB-proximal chromatin microenvironment for DNA repair, but the observed changes in DNA resection point to a role in the regulation of DSB repair pathway choice.

\section{DNA LESIONS ON THE MOVE - AGGREGATION OF DOUBLE-STRAND BREAK IN THREE DIMENSIONS}

Both TAD- and phase separation-associated repair domain formation can be observed at a single DNA lesion. However, the process of $53 \mathrm{BP} 1$-dependent clustering of multiple TAD domains, as well as the inherent biophysical properties of liquid demixing raise the possibility of a higher-order organization of multiple DNA lesions into a single repair "super-focus." Moreover, recent advances in high-resolution live cell microscopy and targeted genome manipulation have uncovered compelling evidence for directed movement of DNA lesions to form aggregates. Although the phenomenon of DSB clustering has been observed in yeast and mammalian cells almost 2 decades ago (Lisby et al., 2003; Aten et al., 2004), until very recently little was known about the underlying mechanistic forces and possible functional relevance. In the following we will discuss novel insight into DSB mobility and one of the pre-eminent nuclear environments they congregate at, the nuclear pore (Figure 3).

\section{Movement and Clustering of DNA Lesions}

Multiple lines of evidence suggest that DSB mobility can be an active process. Almost 10 years ago, homology search of a single DSB in yeast was shown to involve DNA end resection and RAD51-depedent DSB movement (Dion et al., 2012; Miné- 


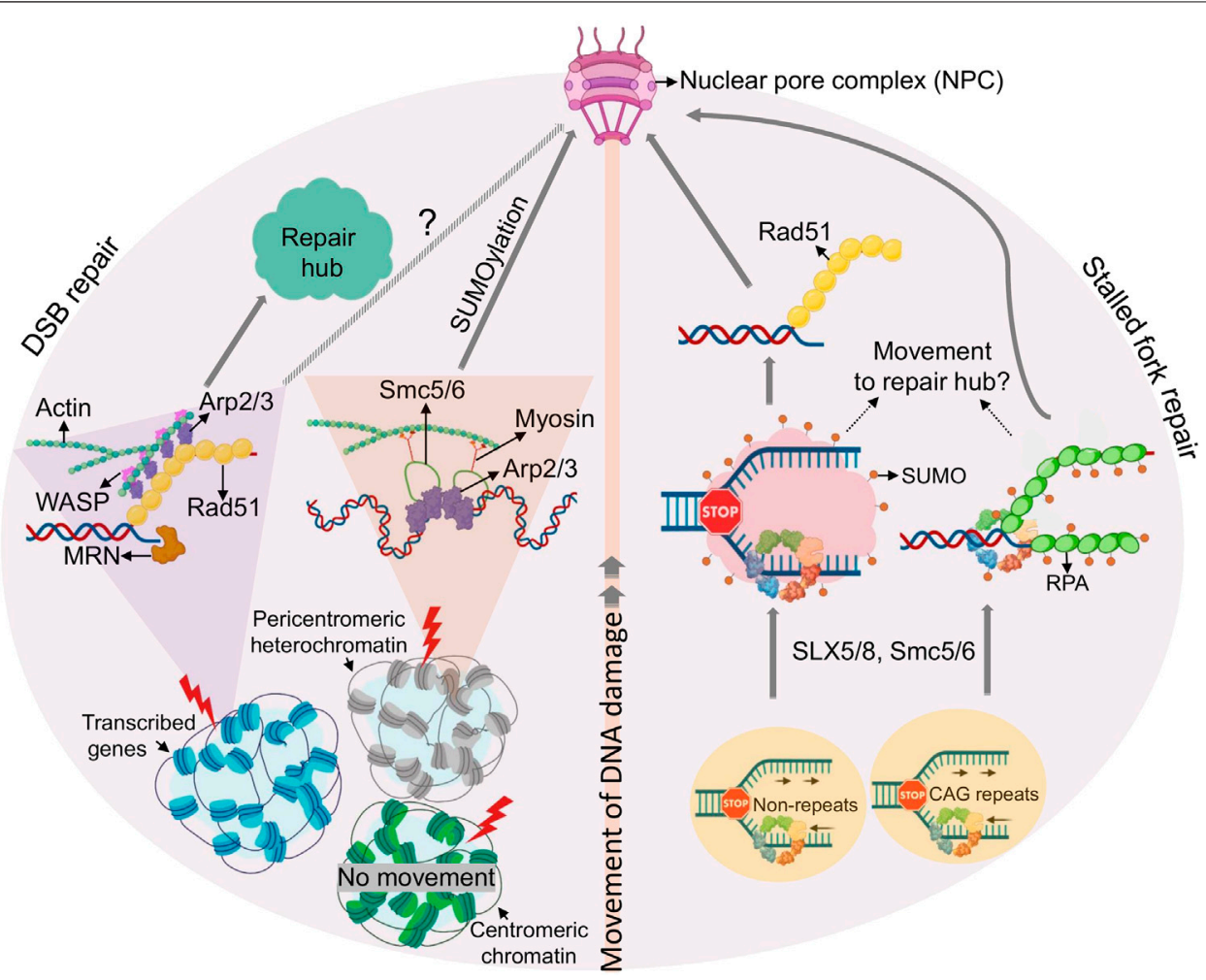

FIGURE 3 | Movement of DSBs and damaged replication forks. Emerging principles of DSB (left) or replication fork mobility (right) are shown. Clockwise from the bottom: Mechanisms of movement are different for DSBs induced in different chromatin environments (see text for details). In brief, DSBs in transcriptionally active chromatin load Rad51 after end resection and move to repair hubs facilitated by actin polymerization. DSBs in pericentromeric heterochromatin initiate resection, but relocate away from hetercochromatin via an SMC5/6 dependent process to facilitate RAD51 loading. Pericentromeric DSBs, and perhaps DSBs within transcribed DSBs regions, are then targeted to the NPC in a manner that involves SUMOylation, myosin, and actin. Counterclockwise from the botton: Stalled replication forks relocate to the NPC in a process involving SUMOylation by Smc5/6 and/or SLX5/8 (shown as red dots). Forks in repetitive sequences are targeted to the NPC via SUMORPA, prior to Rad51 loading, whereas arrested forks at non-repetitive genomic loci are targeted to NPC after RAD51 loading.

Hattab and Rothstein, 2012). RAD51-coated DNA can explore a larger nuclear volume than undamaged DNA, which is thought to facilitate homologous pairing and repair. Mean square displacement (MSD) analyses, which plot the average of the squared distances that a particle has travelled against increasing time intervals, suggested that increased DSB mobility was due to an increase in the radius of confinement, rather than a change in the diffusion coefficient of the damaged locus, pointing to a role for chromatin reorganization in this process (Miné-Hattab and Rothstein, 2012; Hauer and Gasser, 2017). DSB mobility has since then emerged as a complex phenomenon that depends on various factors, including cell cycle phase and DSB location (Kalousi and Soutoglou, 2016; Smith and Rothstein, 2017). Telomeric DSBs, for example, are more mobile than the undamaged chromatin (Dimitrova et al., 2008), whereas UV laser microirradiation or endonucleasemediated induction of DSBs outside of telomeres show limited mobility (Kruhlak et al., 2006; Soutoglou et al., 2007; Roukos et al., 2013). Such discrepancies have sparked intensive efforts to better understand the molecular mechanisms that drive DSB mobility, both in yeast and higher organisms. Much of the initial progress came from studies of broken telomeres, which are relatively easy to monitor in living cells. Telomeric DSBs can result from telomere deprotection and are subject to repair by NHEJ (de Lange, 2018). In mammalian cells, the NHEJ effector 53BP1 was found to promote not only repair but also mobility of broken telomere ends, together with the linker of nucleoskeleton and cytoskeleton (LINC) complex and dynamic microtubules (Lottersberger et al., 2015). 53BP1/LINC-dependent DSB mobility was not limited to telomeres, but was also observed upon irradiation-induced DNA damage. Given the role for 53BP1 in DSB-associated phase separation described in Phase Separation by DNA Repair Factors, it will be interesting to determine if the latter contributes to or complements the mechanic movement forces provided by 53BP1, the LINC complex and microtubules. Precedent for a coordination between phase separation and active movement comes from the observation that RAD52 droplets can cooperate with microtubule filaments to promote DSB clustering and repair (Oshidari et al., 2020). While the functional relevance of 53BP1-mediated DSB clustering remains to be established, this process may help restore proximity of DNA ends that have lost their proper interaction and thereby counteract ectopic repair. However, with increasing DNA damage, aberrant end pairing can 
have fatal outcomes, as evidenced by aberrant telomere end fusions that result in dicentric chromosome formation (Lottersberger et al., 2015).

A distinct type of telomeric break movement has been described in the context of alternative lengthening of telomeres (ALT), an HR-dependent process to maintain telomeres in the absence of telomerase, which is active in $\sim 15 \%$ of cancer types. ALT-associated homologous chromosome synapsis was found to depend on long-range DSB mobility and aggregation into multi-telomere clusters (Cho et al., 2014). Much like HR-prone DSBs in yeast, this process required RAD51, although it further involved the protein dimer Hop1/Mnd1, which also mediates homologous chromosome synapsis during meiosis. Moreover, ALT telomeric DSBs show evidence for directed motion based on MSD analysis (Cho et al., 2014), while DSB movement in yeast was found to be consistent with confined Brownian motion (Dion et al., 2012; Miné-Hattab and Rothstein, 2012). Together, these findings underline the context dependence of telomeric DSB movement.

Telomeres present a unique chromatin environment and the implications of break mobility outside of telomeric regions have only recently been uncovered in vertebrates. In a mass spectrometry approach in Xenopus extracts, Gautier and colleagues identified nuclear actin, the actin-nucleating complex ARP2/3, $\beta$-actin and the ARP2/3 activator WASP as novel, chromatin-associated DSB repair effectors (Schrank et al., 2018). While DNA damage-induced actin polymerization was reported previously (Belin et al., 2015), little was known about its role in DSB repair and/or at broken DNA. Using the mammalian AsiSI endonuclease system described earlier (Aymard et al., 2014), ARP2/3 and WASP were found to preferentially accumulate at HR-prone DSB sites. Consistent with the latter, HR but not NHEJ efficiency was impaired upon inhibition of actin nucleation, or the depletion of WASP or the nucleation factors FORMIN-2 and SPIRE-1/SPIRE-2. Moreover, nuclear actin polymerization was found to be required for G2restricted migration of a subset of DSBs and their aggregation into sub-nuclear clusters. Like mobilty in yeast and at ALT telomeres, AsiSI-induced DSB movement was initiated by DNA end resection (Dion et al., 2012; Miné-Hattab and Rothstein, 2012; Cho et al., 2014; Schrank et al., 2018). Interestingly, ARP2/3 loading was found to enhance end resection and RAD51 loading at AsiSI-induced DSBs in a positive feedback loop (Schrank et al., 2018). DSB movement at HR-prone AsiSI DSBs was further found to be consistent with confined Brownian motion, similar to yeast (Schrank et al., 2018). A role for actin nucleation in the movement of yeast or ALT DSBs remains to be demonstrated.

It should be noted that there is some debate as to when during the cell cycle DSBs cluster. Seemingly in contrast to the findings by the Gautier lab, enhanced DSB clustering was first identified in G1 cells (Aten et al., 2004). Preferential aggregation in G1 was confirmed more recently using Hi-C chromosome conformation capture of AsiSI-dependent DSBs (Aymard et al., 2017), although clustering was similarly restricted to HR-prone break sites. DSB clustering in G1 coincided with delayed DSB repair and was dependent on the MRN complex, FORMIN-2 and the LINC complex, consistent with resection-mediated active movement. Given the identical DSB source (AsiSI), discrepancies in the timing of DSB clustering may reflect distinct experimental readouts, such as the resolution of $\mathrm{Hi}-\mathrm{C}$ versus live cell imaging assays, which is likely to detect significantly smaller aggregates in the case of $\mathrm{Hi}$-C. It will be interesting to determine if distinct "micro" and "macro" aggregate sub-types exist, and how they may differentially contribute to the DDR. One intriguing hypothesis is that transitions in aggregate sub-type may help control repair kinetics during the cell cycle, ensuring HR in S/G2, but preventing $\mathrm{HR}$ in $\mathrm{G} 1$.

Much like HR, actin-mediated DSB mobility is not restricted to transcribed genes. HR in highly repetitive DNA, such as pericentromeric heterochromatin relies on specialized mechanisms to prevent aberrant recombination events. In Drosophila melanogaster, this is achieved by relocalization of DSBs to the nuclear periphery (Chiolo et al., 2011). While proteins responsible for the initial steps of end resection are rapidly recruited within heterochromatin, RAD51 remains excluded, thus preventing homology search and completion of HR. RAD51 loading instead requires resected heterochromatic DSBs to move to the nuclear periphery in a process that involves the SMC5/6 SUMO E3 ligases (Chiolo et al., 2011; Ryu et al., 2015). A similar process has been observed in yeast and at the repetitive $\mathrm{rDNA}$, and has been extensively reviewed elsewhere (Jalal et al., 2017). More recently, Chiolo and colleagues demonstrated that, much like mammalian AsiSI-induced, HRprone DSBs, the movement of heterochromatic breaks to the nuclear periphery in Drosophila requires actin filament formation and the Arp2/3 complex (Caridi et al., 2018). However, while ARP2/3 mediated actin nucleation appears to be sufficient for mobility and clustering of non-heterochromatic DSBs in mammalian cells (Schrank et al., 2018), DSBs within Drosophila heterochromatin further require nuclear myosin, which associates with Smc5/6 proteins to initiate movement (Caridi et al., 2018). Notably, two phases of motion have been described for heterochromatic lesions in Drosophila: confined Brownian motion within the heterochromatin domain, and directed motion towards the NPC, outside of heterochromatin (see Figure 3) (Caridi et al., 2018; Miné-Hattab and Chiolo, 2020). Given that Arp $2 / 3$ promotes non-directed motion of mammalian HR-prone DSBs, actin appears to be able to support both types of motion, implicating additional mobility modulators or species-specific differences. Together, these findings suggest that HR-prone DSBs can initiate movement irrespective of genomic context, but mobility may require additional accessory factors, depending on where the DSBs occur. Why HR-prone breaks move preferentially compared to non-HR prone lesions remains an open question, but further points to a critical role for end resection in this process.

Notably, not all DSBs within compacted chromatin initiate movement, even if they are destined for HR. While DSBs relocalize in the context of pericentromeric heterochromatin as described above, the same does not appear to be true for DSBs in centromeric chromatin, which carries distinct epigenetic marks and occupies distinct nuclear subdomains. The precise nature of this discrepancy remains to be investigated (Tsouroula et al., 
2016). Consistent with the findings by the Gautier lab (Schrank et al., 2018), DSBs in mammalian pericentromeric heterochromatin were found to be positionally stable in G1, where they recruit NHEJ factors, while their resection in S/G2 promoted relocalization away from heterochromatin to allow for RAD51 binding and HR. Centromeric chromatin, on the other hand, was accessible to HR and NHEJ factors throughout the cell cycle and did not require DSB movement for their repair (Tsouroula et al., 2016).

Altogether, these findings add significant new insight into the complexity of the forces that drive DSB mobility, and often DSB clustering, across species, and further place this process at a central position in the control of repair outcome and genome maintenance.

\section{On the Move - But Where to? The Nuclear Pore as a Repair Hub}

Once movement of a DNA lesion is initiated, a common theme across species is its relocalization to the nuclear periphery, and specifically the nuclear pore complex (NPC). Movement of DSBs to the NPC has been extensively reviewed elsewhere (Freudenreich and Su, 2016; Schrank and Gautier, 2019). In the following, we will focus on new insight describing the nuclear pore as a specialized repair microenvironment for aberrant replication forks.

Various studies in yeast have shown that collapsed replication forks localize to the NPC in a process that is reminiscent of the movement of DSBs described in Drosophila (see Movement and Clustering of DNA Lesions, (Chiolo et al., 2011; Ryu et al., 2015)) and similarly depends on SUMO E3 ligases (e.g., SLX5/SLX8, SMC5/6) (Nagai et al., 2008; Freudenreich and Su, 2016; Whalen et al., 2020). Relocation of poly-SUMO-modified arrested forks impedes fork repair by HR until anchorage at the NPC allows for SUMO removal by the SENP SUMO protease Ulp1 and the proteasome, which in turn promotes resumption of DNA synthesis by HR via a process known as Recombination-Dependent Replication (RDR) (Kramarz et al., 2020). Regions undergoing RDR-associated DNA synthesis are prone to chromosomal rearrangements (Lambert et al., 2010; Mizuno et al., 2013), providing a rationale for the spatial segregation of arrested forks within nuclear space.

Relocation of arrested replication forks was observed both at replication obstacles within a unique genomic context and at inherently difficult to replicate repetitive loci. However, the underlying mechanisms appear to be distinct. The relocation of forks collapsed at expanded CAG repeats requires nuclease activities to engage SUMO-RPA onto ssDNA, which prevents Rad51 loading (Whalen et al., 2020). Their anchorage to the NPC is required for RPA removal and efficient Rad51 loading, providing a means to constrain recombination at stalled or collapsed forks until it is required for fork restart. In contrast, relocation of a unique fork block to the NPC was found to occur after RAD51 loading, which may be tolerated due to the less recombinogenic nature of a nonrepetitive DNA (Kramarz et al., 2020). While both relocation events require SLX5/8-mediated SUMOylation, SUMO-RPA accumulation appears to be specific for lesions in repetitive DNA (Whalen et al., 2020). If and how chromatin composition in these distinct genomic contexts determines whether or not RPA is SUMOylated remains to be determined. Altogether, SUMO- based NPC anchorage mechanisms spatially segregate HR events at broken forks at various steps in the repair process, but with the common goal to constrain recombination until it can be safely executed to allow fork restart.

Extending the parallels between the movement of DSBs and stalled or broken forks, recent work by the Cesare lab identified a role for nuclear actin in fork movement to the nuclear periphery (Lamm et al., 2020). Using live and super-resolution imaging, nuclear F-actin was shown to polymerize in response to replication stress in an ATR kinase-dependent manner that further involved WASP and ARP2/3. Much like at heterochromatic DSBs in Drosophila, F-actin and myosin promoted the mobility of stressed replication foci. Actin was further required to resolve replication stress and suppress chromosome and mitotic abnormalities. Finally, nuclear F-actin was detected in human tumor xenografts upon replication stress, indicating disease relevance (Lamm et al., 2020; Lamm et al., 2021). Beyond the response to replication stress, actin dynamics were recently shown to facilitate replication initiation in unperturbed cells by promoting the loading of cyclindependent kinase $(\mathrm{CDK})$ and proliferating cell nuclear antigen (PCNA) onto chromatin (Parisis et al., 2017). If the latter contributes to replication re-initiation at stalled forks upon their F-actin-dependent relocalization remains to be investigated.

Not surprisingly, difficult-to-replicate ALT telomeric DNA also localized to the nuclear periphery in an actinpolymerization-dependent manner in response to replication stress (Lamm et al., 2020). Similarly, in yeast, subtelomeric DSBs were found to move to the nuclear pore for repair via break-induced replication, a means to repair single-ended DSBs often associated with the ALT pathways (Chung et al., 2015; Dilley et al., 2016; Oshidari et al., 2018). Movement of subtelomeric breaks require kinesin motor proteins and microtubule polymerization, extending the repertoire of motor proteins at stalled forks beyond F-actin (Chung et al., 2015; Oshidari et al., 2018). Of note, unlike stalled replication forks, endonuclease-mediated DSBs at subtelomeric regions in ALT cells were shown to aggregate predominantly in ALTassociated PML nuclear bodies, which may promote clustering and recombination of telomere ends (Cho et al., 2014) (see also Movement and Clustering of DNA Lesions). It will be interesting to determine what accounts for the differential targeting of these distinct, ALT telomere-associated DNA lesions.

Altogether, these recent advances highlight the importance of the nuclear pore as a repair-permissive microenvironment that supports the resolution of both DSBs and replication stress. Future work will need to uncover why the NPC presents a preferential "meeting point" for DNA lesions, and why this environment appears to be selectively associated with HR.

\section{D GENOME ORGANIZATION-RELATED GENOME INSTABILITY IN CANCER AND DISEASE}

Having reviewed the importance of 3D nuclear organization in the context of genome maintenance and accurate DNA 
A

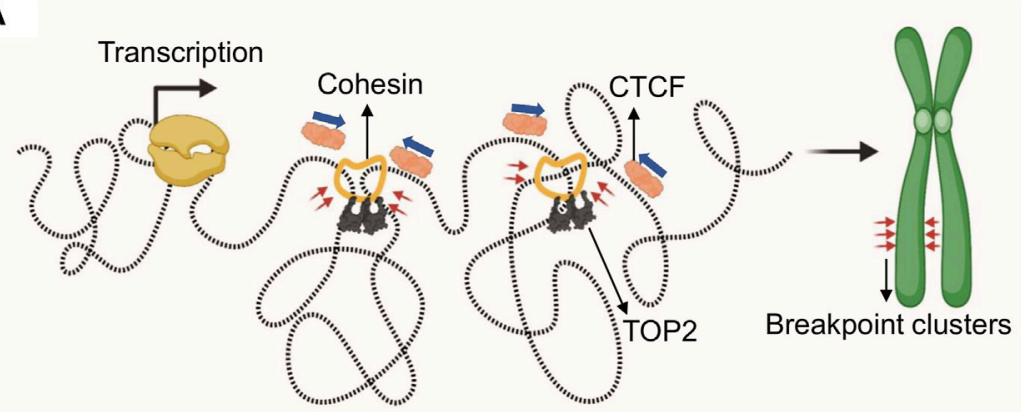

B

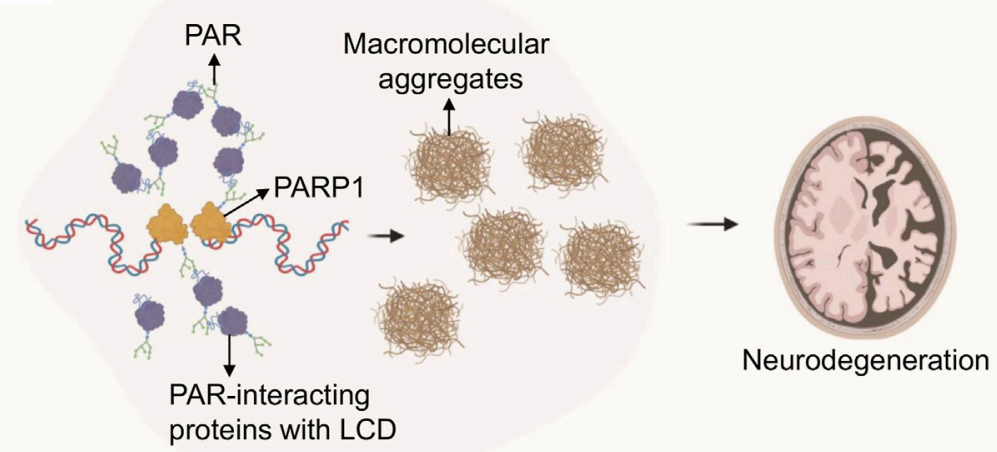

FIGURE 4 | Role of genome architecture in genome instability and disease. (A) Loop extrusion by cohesin creates tortional stress on DNA, which is relieved by topoisomerases. TOP2 inhibition via etoposide covalently traps TOP2 at TAD boundaries which generates DSBs in the presence of transcription, ultimately resulting in chromosomal translocations. Etoposide-treatment in cancer is frequently associated with recurrent chromosomal translocations at TAD-boundary-associated breakpoint clusters. (B) DNA damage-driven, protein-rich biomolecular condensates are linked to neurodegeneration in A-T or ATLD patients. DDR defects in these patients cause PARP1 hyperactivation and PAR chain accumulation. PAR-dependent protein aggregates are found in A-T patient cerebellum.

repair, it must be noted that the spatial arrangement of chromatin has a direct effect on genome instability, often dictating the outcome of translocations and other aberrant repair events (Hakim et al., 2012; Roukos et al., 2013; Roukos and Misteli, 2014). In the following, we will discuss how nuclear topology can affect mutagenesis and the development of disease.

\section{Topologically Associated Domains, DNA Double-Strand Breaks and Genome Instability}

First experimental evidence that TADs, and particularly the anchor regions of these chromosome loops, may pose a threat to genome integrity came from the Nussenzweig lab in 2017 (Canela et al., 2017). Loop anchor regions, as defined by both Hi$\mathrm{C}$ contact maps and ChIP for the anchoring factors CTCF and cohesin (RAD21), were found to be a hot spot for Topoisomerase 2 (TOP2)-mediated DSB breakage. Consistent with this, TOP2B, one of two TOP2 isoforms in mammalian cells, has been shown to physically interact with CTCF and cohesin (Witcher and Emerson, 2009; Uusküla-Reimand et al., 2016) and is enriched in CTCF/cohesin-bound genomic regions (Madabhushi et al., 2015; Uusküla-Reimand et al., 2016). Of note, CTCF/TOP2associated DSBs at TAD boundaries frequently involve breakpoint clusters that are commonly translocated in cancer, and were shown to drive cell-type- and tumor-specific chromosomal translocations (Canela et al., 2019; Gothe et al., 2019). Thus, loop anchors appear to be genomic fragile sites that can generate DSBs and chromosomal rearrangements. Moreover, these regions are particularly sensitive to treatment with the TOP2 poison and chemotherapeutic agent etoposide, which stabilizes the TOP2 cleavage complex and thus enhances DSB formation. As a result, etoposide treatment is frequently associated with recurrent chromosome translocations involving TAD boundaries in therapy-related myeloid leukemias (t-AML) (Wright and Vaughan, 2014).

While TOP2 chromatin localization and trapping at CTCF/ cohesion anchors was shown to be independent of transcription (Canela et al., 2017), the conversion of trapped TOP2 cleavage complexes into DSBs correlates with transcriptional output and directionality (Canela et al., 2019; Gothe et al., 2019). Consistent with the latter, genes that recurrently translocate to drive leukemias are highly transcribed and are enriched at loop 
anchors (Gothe et al., 2019). Transcription and 3D chromosome folding thus pose a joint topological threat to genomic stability and are key contributors to the occurrence of genome rearrangements that drive cancer (Canela et al., 2019; Gothe et al., 2019) (Figure 4A).

In addition to topoisomerase poisons, mild replication stress was also able to trigger DNA fragility at TAD boundaries, often mapping to difficult to replicate genomic regions known as common fragile sites (CFSs) (Sarni et al., 2020). This effect was particularly pronounced at transcribed large genes that span TAD boundaries and coincided with a delay in replication timing (Sarni et al., 2020). Of note, replication domain boundaries overlap TAD boundaries, suggesting that TADs are regulatory units of replication timing (Pope et al., 2014; Marchal et al., 2019), and providing a rationale for the unique sensitivity of $\mathrm{TAD}$ boundaries to replication delays. It will be interesting to determine if this process further involves TOP2-mediated DSB induction.

\section{Phase Separation in Neurodegenerative Disease}

While DNA lesion-associated phase separation is emerging as an integral and dynamic aspect of the DNA damage response, recent work by Paull and colleagues suggests that PAR-seeded liquid demixing may ultimately result in insoluble protein-rich biomolecular condensates observed in the cerebellar neurodegenerative disorder associated with ataxia-telangiectasia (A-T) (Lee et al., 2021) (Figure 4B). A-T is caused by the loss of ATM kinase, and hypomorphic mutations in the MRE11 repair factor can cause the related A-T-like disorder (ATLD), implicating a prominent role for DNA damage in disease progression (Taylor et al., 2004; Regal et al., 2013). While malignancy and immunodeficiency of A-T and ATLD patients is readily explained by DNA repair defects, the source of neurotoxicity in these patients remains poorly understood (Shiloh, 2020). Genetic ATM separation-of-function mutations previously demonstrated that ATM mutations associated with a loss of its activation by oxidative damage resulted in widespread protein aggregation (Guo et al., 2010; Lee et al., 2018). In seeking to understand the molecular basis for ATM function in protein homeostasis, the Paull lab identified a central role for PARP-dependent nuclear condensates arising from intrinsically disordered proteins associating with PARylated genomic sites (Lee et al., 2021). PARP activation in ATM deficient cells was shown to depend on increased oxidative stress, which in turn caused transcriptionassociated damage, RNA:DNA hybrid formation and ssDNA lesions. Of note, PARP activation was found to occur independently of oxidative lesions in ATLD patients, suggesting that alternative mechanisms exist to initiate PAR-dependent protein aggregation in ATLD. Of relevance for neurodegenerative disease, PAR-related protein-rich condensates were found to be wide-spread in A-T patient cerebellum (Lee et al., 2021). These findings point to an inherent danger of DNA damageassociated phase separation, particularly in the presence of excessive DNA damage or repair defects that may prevent its dynamic formation and resolution. It will be interesting to determine if other repair-defect- and/or RNA:DNA hybrid- associated neurodegenerative diseases exhibit similar pathology (Moreira et al., 2004; Loomis et al., 2014; Perego et al., 2019).

\section{PERSPECTIVE}

Three-dimensional nuclear organization is central to both accurate and aberrant genome maintenance. Continued efforts to map dynamic 3D changes in nuclear space in response to perturbations such as DNA damage, transcription or replication, are critical to advance our understanding of the pathways and factors that control genome maintenance. Recent advances in characterizing the mammalian nucleus in space and time, such as the NIH 4D Nucleome or the ENCODE projects (Consortium, 2004; Dekker et al., 2017), are providing relevant, high-resolution technologies and insight to inform future research in DNA repair. Some of the emerging issues in the field have been indicated throughout this review, but we would like to highlight a few key aspects we consider integral moving forward.

First, how does active movement of DSBs relate to the biophysical separation of repair environments via liquid demixing? Are these events part of the same process, or is there a choice between one and the other, and what would that choice depend on? Can we exploit aggregation and mobility mechanisms to manipulate repair processes, outcome and overall genome maintenance? The latter is supported by intriguing findings that inhibition of actin nucleation can sensitize cancer cells to both PARP inhibition and the DNA polymerase inhibitor aphidicolin (Schrank et al., 2018). Conversely, damage-induced liquid demixing appears to contribute to protein condensates associated with neurodegenerative disorders (Lee et al., 2021), while a potentially beneficial impact of phase separation on DNA repair reactions and concomitant genome maintenance remains to be identified.

Second, the aggregation of multiple DSBs within nuclear space, or at specialized micro-environments such as the NPC, begs the question of why distinct DSBs need to be brought together during repair. At first glance, this seems to be a dangerous proposition, as it may facilitate illegitimate repair events. Indeed, telomere fusions and dicentric chromosome formation are thought to be a result of this process (Lottersberger et al., 2015). What, then, are the benefits of specialized repair micro-environments? Or are DSB clusters merely a natural consequence of a condensation process that evolved to locally increase the concentration of repair factors? And what is the composition of phase separated condensates, HR-associated F-actin-dependent DSB aggregates and DNA lesion-associated NPCs? A better molecular and structural understanding of these specialized repair environments will no doubt help us determine their role in the repair process.

Third, although we may finally understand what leads to often Mb-sized DSB repair foci (Arnould et al., 2021), it remains a mystery as to why DSB marks such as $\gamma \mathrm{H} 2 \mathrm{AX}$ and its downstream effector proteins need to cover the extent of DNA they do. Placing these findings in the context of TADs will likely provide additional insight, but more work is needed to understand this most basic feature of DSB repair. Despite 
these remaining challenges, however, our understanding of DSB repair has advanced significantly with the consideration of a third nuclear dimension, as well as the complex arrangements of DNA lesions within nuclear space. We look forward to seeing this insight translated into actionable means to manipulate DNA repair to prevent or treat diseases associated with genome maintenance defects.

\section{AUTHOR CONTRIBUTIONS}

$\mathrm{PO}$ and RS wrote the manuscript with help from MIA. RS designed the figures using BioRender.com with guidance from PO.

\section{REFERENCES}

Alabert, C., Jasencakova, Z., and Groth, A. (2017). Chromatin Replication and Histone Dynamics. Adv. Exp. Med. Biol. 1042, 311-333. doi:10.1007/978-98110-6955-0_15

Aladjem, M. I., and Redon, C. E. (2017). Order from Clutter: Selective Interactions at Mammalian Replication Origins. Nat. Rev. Genet. 18 (2), 101-116. doi:10.1038/nrg.2016.141

Alagoz, M., Katsuki, Y., Ogiwara, H., Ogi, T., Shibata, A., Kakarougkas, A., et al. (2015). SETDB1, HP1 and SUV39 Promote Repositioning of 53BP1 to Extend Resection during Homologous Recombination in G2 Cells. Nucleic Acids Res. 43 (16), 7931-7944. doi:10.1093/nar/gkv722

Altmeyer, M., Neelsen, K. J., Teloni, F., Pozdnyakova, I., Pellegrino, S., Grøfte, M., et al. (2015). Liquid Demixing of Intrinsically Disordered Proteins Is Seeded by poly(ADP-Ribose). Nat. Commun. 6, 8088. doi:10.1038/ ncomms 9088

Arnould, C., Rocher, V., Finoux, A.-L., Clouaire, T., Li, K., Zhou, F., et al. (2021). Loop Extrusion as a Mechanism for Formation of DNA Damage Repair Foci. Nature 590 (7847), 660-665. doi:10.1038/s41586-021-03193-z

Aten, J. A., Stap, J., Krawczyk, P. M., van Oven, C. H., Hoebe, R. A., Essers, J., et al. (2004). Dynamics of DNA Double-Strand Breaks Revealed by Clustering of Damaged Chromosome Domains. Science 303 (5654), 92-95. doi:10.1126/ science. 1088845

Aumiller, W. M., Jr., Pir Cakmak, F., Davis, B. W., and Keating, C. D. (2016). RNAbased Coacervates as a Model for Membraneless Organelles: Formation, Properties, and Interfacial Liposome Assembly. Langmuir 32 (39), 10042-10053. doi:10.1021/acs.langmuir.6b02499

Aymard, F., Aguirrebengoa, M., Guillou, E., Javierre, B. M., Bugler, B., Arnould, C., et al. (2017). Genome-wide Mapping of Long-Range Contacts Unveils Clustering of DNA Double-Strand Breaks at Damaged Active Genes. Nat. Struct. Mol. Biol. 24 (4), 353-361. doi:10.1038/nsmb.3387

Aymard, F., Bugler, B., Schmidt, C. K., Guillou, E., Caron, P., Briois, S., et al. (2014). Transcriptionally Active Chromatin Recruits Homologous Recombination at DNA Double-Strand Breaks. Nat. Struct. Mol. Biol. 21 (4), 366-374. doi:10.1038/nsmb.2796

Ba, Z., Lou, J., Ye, A. Y., Dai, H.-Q., Dring, E. W., Lin, S. G., et al. (2020). CTCF Orchestrates Long-Range Cohesin-Driven V(D)J Recombinational Scanning. Nature 586 (7828), 305-310. doi:10.1038/s41586-020-2578-0

Banani, S. F., Lee, H. O., Hyman, A. A., and Rosen, M. K. (2017). Biomolecular Condensates: Organizers of Cellular Biochemistry. Nat. Rev. Mol. Cel Biol 18 (5), 285-298. doi:10.1038/nrm.2017.7

Belin, B. J., Lee, T., and Mullins, R. D. (2015). DNA Damage Induces Nuclear Actin Filament Assembly by Formin -2 and Spire-1/2 that Promotes Efficient DNA Repair. [corrected]. Elife 4, e07735. doi:10.7554/eLife.07735

Bellush, J. M., and Whitehouse, I. (2017). DNA Replication through a Chromatin Environment. Philos. Trans. R. Soc. Lond. B Biol. Sci. 372 (1731), 20160287. doi:10.1098/rstb.2016.0287

Bickmore, W. A. (2013). The Spatial Organization of the Human Genome. Annu. Rev. Genom. Hum. Genet. 14, 67-84. doi:10.1146/annurev-genom-091212153515

\section{FUNDING}

This work was supported by the Intramural Research Program of the NIH, NCI, Center for Cancer Research (ZIA BC010411 to MA).

\section{ACKNOWLEDGMENTS}

We apologize to the many authors who's work we were unable to cite due to space constraints. We thank M. Seidman for critical reading of the manuscript. MA is supported by the Intramural Research Program of the NIH, NCI, Center for Cancer Research (ZIA BC010411).

Boeynaems, S., Alberti, S., Fawzi, N. L., Mittag, T., Polymenidou, M., Rousseau, F., et al. (2018). Protein Phase Separation: A New Phase in Cell Biology. Trends Cel Biol. 28 (6), 420-435. doi:10.1016/j.tcb.2018.02.004

Brangwynne, C. P., Eckmann, C. R., Courson, D. S., Rybarska, A., Hoege, C., Gharakhani, J., et al. (2009). Germline P Granules Are Liquid Droplets that Localize by Controlled Dissolution/condensation. Science 324 (5935), 1729-1732. doi:10.1126/science. 1172046

Britton, S., Dernoncourt, E., Delteil, C., Froment, C., Schiltz, O., Salles, B., et al. (2014). DNA Damage Triggers SAF-A and RNA Biogenesis Factors Exclusion from Chromatin Coupled to R-Loops Removal. Nucleic Acids Res. 42 (14), 9047-9062. doi:10.1093/nar/gku601

Canela, A., Maman, Y., Huang, S.-y. N., Wutz, G., Tang, W., Zagnoli-Vieira, G., et al. (2019). Topoisomerase II-Induced Chromosome Breakage and Translocation Is Determined by Chromosome Architecture and Transcriptional Activity. Mol. Cel 75 (2), 252-266. e8. doi:10.1016/ j.molcel.2019.04.030

Canela, A., Maman, Y., Jung, S., Wong, N., Callen, E., Day, A., et al. (2017). Genome Organization Drives Chromosome Fragility. Cell 170 (3), 507-521. e18. doi:10.1016/j.cell.2017.06.034

Caridi, C. P., D’Agostino, C., Ryu, T., Zapotoczny, G., Delabaere, L., Li, X., et al. (2018). Nuclear F-Actin and Myosins Drive Relocalization of Heterochromatic Breaks. Nature 559 (7712), 54-60. doi:10.1038/s41586-018-0242-8

Caron, P., Aymard, F., Iacovoni, J. S., Briois, S., Canitrot, Y., Bugler, B., et al. (2012). Cohesin Protects Genes against $\gamma \mathrm{H} 2 \mathrm{AX}$ Induced by DNA Double-Strand Breaks. Plos Genet. 8 (1), e1002460. doi:10.1371/journal.pgen.1002460

Catucci, I., Osorio, A., Arver, B., Neidhardt, G., Bogliolo, M., Zanardi, F., et al. (2018). Individuals with FANCM Biallelic Mutations Do Not Develop Fanconi Anemia, but Show Risk for Breast Cancer, Chemotherapy Toxicity and May Display Chromosome Fragility. Genet. Med. 20 (4), 452-457. doi:10.1038/ gim.2017.123

Chang, H. H. Y., Pannunzio, N. R., Adachi, N., and Lieber, M. R. (2017). Nonhomologous DNA End Joining and Alternative Pathways to Double-Strand Break Repair. Nat. Rev. Mol. Cel Biol 18 (8), 495-506. doi:10.1038/nrm.2017.48

Chen, H., Ruiz, P. D., Novikov, L., Casill, A. D., Park, J. W., and Gamble, M. J. (2014). MacroH2A1.1 and PARP-1 Cooperate to Regulate Transcription by Promoting CBP-Mediated H2B Acetylation. Nat. Struct. Mol. Biol. 21 (11), 981-989. doi:10.1038/nsmb.2903

Chiolo, I., Minoda, A., Colmenares, S. U., Polyzos, A., Costes, S. V., and Karpen, G. H. (2011). Double-strand Breaks in Heterochromatin Move outside of a Dynamic HPla Domain to Complete Recombinational Repair. Cell 144 (5), 732-744. doi:10.1016/j.cell.2011.02.012

Cho, N. W., Dilley, R. L., Lampson, M. A., and Greenberg, R. A. (2014). Interchromosomal Homology Searches Drive Directional ALT Telomere Movement and Synapsis. Cell 159 (1), 108-121. doi:10.1016/j.cell.2014.08.030

Cho, W.-K., Spille, J.-H., Hecht, M., Lee, C., Li, C., Grube, V., et al. (2018). Mediator and RNA Polymerase II Clusters Associate in Transcription-dependent Condensates. Science 361 (6400), 412-415. doi:10.1126/science.aar4199

Chung, D. K. C., Chan, J. N. Y., Strecker, J., Zhang, W., Ebrahimi-Ardebili, S., Lu, T., et al. (2015). Perinuclear Tethers License Telomeric DSBs for a Broad Kinesin- and NPC-dependent DNA Repair Process. Nat. Commun. 6, 7742. doi: $10.1038 /$ ncomms 8742 
Consortium, E. P. (2004). The ENCODE (ENCyclopedia of DNA Elements) Project. Science 306 (5696), 636-640. doi:10.1126/science.1105136

Consortium, E. P., Moore, J. E., Purcaro, M. J., Pratt, H. E., Epstein, C. B., Shoresh, N., et al. (2020). Expanded Encyclopaedias of DNA Elements in the Human and Mouse Genomes. Nature 583 (7818), 699-710. doi:10.1038/ s41586-020-2493-4

Covo, S., Westmoreland, J. W., Gordenin, D. A., and Resnick, M. A. (2010). Cohesin Is Limiting for the Suppression of DNA Damage-Induced Recombination between Homologous Chromosomes. Plos Genet. 6 (7), e1001006. doi:10.1371/journal.pgen.1001006

Dabin, J., Fortuny, A., and Polo, S. E. (2016). Epigenome Maintenance in Response to DNA Damage. Mol. Cel 62 (5), 712-727. doi:10.1016/ j.molcel.2016.04.006

Dai, H.-Q., Hu, H., Lou, J., Ye, A. Y., Ba, Z., Zhang, X., et al. (2021). Loop Extrusion Mediates Physiological Igh Locus Contraction for RAG Scanning. Nature 590 (7845), 338-343. doi:10.1038/s41586-020-03121-7

Davidson, I. F., Bauer, B., Goetz, D., Tang, W., Wutz, G., and Peters, J.-M. (2019). DNA Loop Extrusion by Human Cohesin. Science 366 (6471), 1338-1345. doi:10.1126/science.aaz3418

Davidson, I. F., and Peters, J.-M. (2021). Genome Folding through Loop Extrusion by SMC Complexes. Nat. Rev. Mol. Cel Biol 22 (7), 445-464. doi:10.1038/ s41580-021-00349-7

de Lange, T. (2018). Shelterin-Mediated Telomere Protection. Annu. Rev. Genet. 52, 223-247. doi:10.1146/annurev-genet-032918-021921

Dekker, J., Belmont, A. S., Guttman, M., Leshyk, V. O., Lis, J. T., Lomvardas, S., et al. (2017). The 4D Nucleome Project. Nature 549 (7671), 219-226. doi:10.1038/nature23884

Dekker, J., and Mirny, L. (2016). The 3D Genome as Moderator of Chromosomal Communication. Cell 164 (6), 1110-1121. doi:10.1016/j.cell.2016.02.007

Dilley, R. L., Verma, P., Cho, N. W., Winters, H. D., Wondisford, A. R., and Greenberg, R. A. (2016). Break-induced Telomere Synthesis Underlies Alternative Telomere Maintenance. Nature 539 (7627), 54-58. doi:10.1038/nature20099

Dimitrova, N., Chen, Y.-C. M., Spector, D. L., and de Lange, T. (2008). 53BP1 Promotes Non-homologous End Joining of Telomeres by Increasing Chromatin Mobility. Nature 456 (7221), 524-528. doi:10.1038/nature07433

Dion, V., Kalck, V., Horigome, C., Towbin, B. D., and Gasser, S. M. (2012). Increased Mobility of Double-Strand Breaks Requires Mec1, Rad9 and the Homologous Recombination Machinery. Nat. Cel Biol 14 (5), 502-509. doi:10.1038/ncb2465

Erdel, F., Rademacher, A., Vlijm, R., Tünnermann, J., Frank, L., Weinmann, R., et al. (2020). Mouse Heterochromatin Adopts Digital Compaction States without Showing Hallmarks of HP1-Driven Liquid-Liquid Phase Separation. Mol. Cel 78 (2), 236-249. e7. doi:10.1016/j.molcel.2020.02.005

Erdel, F., and Rippe, K. (2018). Formation of Chromatin Subcompartments by Phase Separation. Biophysical J. 114 (10), 2262-2270. doi:10.1016/ j.bpj.2018.03.011

Ferrand, J., Plessier, A., and Polo, S. E. (2021). Control of the Chromatin Response to DNA Damage: Histone Proteins Pull the Strings. Semin. Cel Develop. Biol. 113, 75-87. doi:10.1016/j.semcdb.2020.07.002

Finn, E. H., Pegoraro, G., Brandão, H. B., Valton, A.-L., Oomen, M. E., Dekker, J., et al. (2019). Extensive Heterogeneity and Intrinsic Variation in Spatial Genome Organization. Cell 176 (6), 1502-1515. doi:10.1016/j.cell.2019.01.020

Francia, S., Michelini, F., Saxena, A., Tang, D., de Hoon, M., Anelli, V., et al. (2012). Site-specific DICER and DROSHA RNA Products Control the DNA Damage Response. Nature 488 (7410), 231-235. doi:10.1038/nature11179

Frattini, C., Promonet, A., Alghoul, E., Vidal-Eychenie, S., Lamarque, M., Blanchard, M.-P., et al. (2021). TopBP1 Assembles Nuclear Condensates to Switch on ATR Signaling. Mol. Cel 81 (6), 1231-1245. e8. doi:10.1016/ j.molcel.2020.12.049

Freudenreich, C. H., and Su, X. A. (2016). Relocalization of DNA Lesions to the Nuclear Pore Complex. FEMS Yeast Res. 16 (8). doi:10.1093/femsyr/fow095

Fudenberg, G., Abdennur, N., Imakaev, M., Goloborodko, A., and Mirny, L. A. (2017). Emerging Evidence of Chromosome Folding by Loop Extrusion. Cold Spring Harb Symp. Quant Biol. 82, 45-55. doi:10.1101/ sqb.2017.82.034710

Ganji, M., Shaltiel, I. A., Bisht, S., Kim, E., Kalichava, A., Haering, C. H., et al. (2018). Real-time Imaging of DNA Loop Extrusion by Condensin. Science 360 (6384), 102-105. doi:10.1126/science.aar7831
Gibson, B. A., Doolittle, L. K., Schneider, M. W. G., Jensen, L. E., Gamarra, N., Henry, L., et al. (2019). Organization of Chromatin by Intrinsic and Regulated Phase Separation. Cell 179 (2), 470-484. e21. doi:10.1016/j.cell.2019.08.037

Gothe, H. J., Bouwman, B. A. M., Gusmao, E. G., Piccinno, R., Petrosino, G., Sayols, S., et al. (2019). Spatial Chromosome Folding and Active Transcription Drive DNA Fragility and Formation of Oncogenic MLL Translocations. Mol. Cel 75 (2), 267-283. e12. doi:10.1016/j.molcel.2019.05.015

Guo, Q., Shi, X., and Wang, X. (2021). RNA and Liquid-Liquid Phase Separation. Non-coding RNA Res. 6 (2), 92-99. doi:10.1016/j.ncrna.2021.04.003

Guo, Z., Kozlov, S., Lavin, M. F., Person, M. D., and Paull, T. T. (2010). ATM Activation by Oxidative Stress. Science 330 (6003), 517-521. doi:10.1126/ science. 1192912

Hakim, O., Resch, W., Yamane, A., Klein, I., Kieffer-Kwon, K.-R., Jankovic, M., et al. (2012). DNA Damage Defines Sites of Recurrent Chromosomal Translocations in B Lymphocytes. Nature 484 (7392), 69-74. doi:10.1038/nature10909

Handwerger, K. E., Cordero, J. A., and Gall, J. G. (2005). Cajal Bodies, Nucleoli, and Speckles in the Xenopus Oocyte Nucleus Have a Low-Density, Sponge-like Structure. MBoC 16 (1), 202-211. doi:10.1091/mbc.e04-08-0742

Hansen, A. S., Cattoglio, C., Darzacq, X., and Tjian, R. (2018). Recent Evidence that TADs and Chromatin Loops Are Dynamic Structures. Nucleus 9 (1), 20-32. doi:10.1080/19491034.2017.1389365

Harrigan, J. A., Belotserkovskaya, R., Coates, J., Dimitrova, D. S., Polo, S. E., Bradshaw, C. R., et al. (2011). Replication Stress Induces 53BP1-Containing OPT Domains in G1 Cells. J. Cel Biol 193 (1), 97-108. doi:10.1083/ jcb.201011083

Hauer, M. H., and Gasser, S. M. (2017). Chromatin and Nucleosome Dynamics in DNA Damage and Repair. Genes Dev. 31 (22), 2204-2221. doi:10.1101/ gad.307702.117

Hnisz, D., Shrinivas, K., Young, R. A., Chakraborty, A. K., and Sharp, P. A. (2017). A Phase Separation Model for Transcriptional Control. Cell 169 (1), 13-23. doi:10.1016/j.cell.2017.02.007

Hustedt, N., and Durocher, D. (2016). The Control of DNA Repair by the Cell Cycle. Nat. Cel Biol 19 (1), 1-9. doi:10.1038/ncb3452

Iacovoni, J. S., Caron, P., Lassadi, I., Nicolas, E., Massip, L., Trouche, D., et al. (2010). High-resolution Profiling of $\gamma \mathrm{H} 2 \mathrm{AX}$ Around DNA Double Strand Breaks in the Mammalian Genome. EMBO J. 29 (8), 1446-1457. doi:10.1038/emboj.2010.38

Jalal, D., Chalissery, J., and Hassan, A. H. (2017). Genome Maintenance in Saccharomyces cerevisiae: the Role of SUMO and SUMO-Targeted Ubiquitin Ligases. Nucleic Acids Res. 45 (5), 2242-2261. doi:10.1093/nar/gkw1369

Janssen, A., Colmenares, S. U., and Karpen, G. H. (2018). Heterochromatin: Guardian of the Genome. Annu. Rev. Cel Dev. Biol. 34, 265-288. doi:10.1146/annurev-cellbio-100617-062653

Jasin, M., and Rothstein, R. (2013). Repair of Strand Breaks by Homologous Recombination. Cold Spring Harbor Perspect. Biol. 5 (11), a012740. doi:10.1101/ cshperspect.a012740

Kalousi, A., and Soutoglou, E. (2016). Nuclear Compartmentalization of DNA Repair. Curr. Opin. Genet. Develop. 37, 148-157. doi:10.1016/j.gde.2016.05.013

Karakashev, S., Fukumoto, T., Zhao, B., Lin, J., Wu, S., Fatkhutdinov, N., et al. (2020). EZH2 Inhibition Sensitizes CARM1-High, Homologous Recombination Proficient Ovarian Cancers to PARP Inhibition. Cancer Cell 37 (2), 157-167. doi:10.1016/j.ccell.2019.12.015

Kempfer, R., and Pombo, A. (2020). Methods for Mapping 3D Chromosome Architecture. Nat. Rev. Genet. 21 (4), 207-226. doi:10.1038/s41576-019-0195-2

Khurana, S., Kruhlak, M. J., Kim, J., Tran, A. D., Liu, J., Nyswaner, K., et al. (2014). A Macrohistone Variant Links Dynamic Chromatin Compaction to BRCA1dependent Genome Maintenance. Cel Rep. 8 (4), 1049-1062. doi:10.1016/ j.celrep.2014.07.024

Kilic, S., Lezaja, A., Gatti, M., Bianco, E., Michelena, J., Imhof, R., et al. (2019). Phase Separation of 53BP1 Determines Liquid-like Behavior of DNA Repair Compartments. EMBO J. 38 (16), e101379. doi:10.15252/embj.2018101379

Kim, J., Sturgill, D., Sebastian, R., Khurana, S., Tran, A. D., Edwards, G. B., et al. (2018). Replication Stress Shapes a Protective Chromatin Environment across Fragile Genomic Regions. Mol. Cel 69 (1), 36-47. e7. doi:10.1016/ j.molcel.2017.11.021

Klein, K. N., Zhao, P. A., Lyu, X., Sasaki, T., Bartlett, D. A., Singh, A. M., et al. (2021). Replication Timing Maintains the Global Epigenetic State in Human Cells. Science 372 (6540), 371-378. doi:10.1126/science.aba5545 
Kramarz, K., Schirmeisen, K., Boucherit, V., Ait Saada, A., Lovo, C., Palancade, B., et al. (2020). The Nuclear Pore Primes Recombination-dependent DNA Synthesis at Arrested forks by Promoting SUMO Removal. Nat. Commun. 11 (1), 5643. doi:10.1038/s41467-020-19516-z

Kraus, W. L. (2020). PARPs and ADP-Ribosylation: 60 Years on. Genes Dev. 34, 251-253. doi:10.1101/gad.336420.120

Kruhlak, M. J., Celeste, A., Dellaire, G., Fernandez-Capetillo, O., Muiller, W. G., McNally, J. G., et al. (2006). Changes in Chromatin Structure and Mobility in Living Cells at Sites of DNA Double-Strand Breaks. J. Cel Biol 172 (6), 823-834. doi:10.1083/jcb.200510015

Lambert, S., Mizuno, K. i., Blaisonneau, J., Martineau, S., Chanet, R., Fréon, K., et al. (2010). Homologous Recombination Restarts Blocked Replication forks at the Expense of Genome Rearrangements by Template Exchange. Mol. Cel 39 (3), 346-359. doi:10.1016/j.molcel.2010.07.015

Lamm, N., Read, M. N., Nobis, M., Van Ly, D., Page, S. G., Masamsetti, V. P., et al. (2020). Nuclear F-Actin Counteracts Nuclear Deformation and Promotes fork Repair during Replication Stress. Nat. Cel Biol 22 (12), 1460-1470. doi:10.1038/ s41556-020-00605-6

Lamm, N., Rogers, S., and Cesare, A. J. (2021). Chromatin Mobility and Relocation in DNA Repair. Trends Cel Biol 31, 843-855. doi:10.1016/j.tcb.2021.06.002

Langdon, E. M., Qiu, Y., Ghanbari Niaki, A., McLaughlin, G. A., Weidmann, C. A., Gerbich, T. M., et al. (2018). mRNA Structure Determines Specificity of a polyQDriven Phase Separation. Science 360 (6391), 922-927. doi:10.1126/science.aar7432

Larson, A. G., Elnatan, D., Keenen, M. M., Trnka, M. J., Johnston, J. B., Burlingame, A. L., et al. (2017). Liquid Droplet Formation by HP1a Suggests a Role for Phase Separation in Heterochromatin. Nature 547 (7662), 236-240. doi:10.1038/nature22822

Larson, A. G., and Narlikar, G. J. (2018). The Role of Phase Separation in Heterochromatin Formation, Function, and Regulation. Biochemistry 57 (17), 2540-2548. doi:10.1021/acs.biochem.8b00401

Lebeaupin, T., Timinszky, Gyula., Timinszky, H. G., and Huet, S. (2015). Chromatin Dynamics at DNA Breaks: what, How and Why? AIMS Biophys. 2 (4), 458-475. doi:10.3934/biophy.2015.4.458

Lee, J.-H., Ryu, S. W., Ender, N. A., and Paull, T. T. (2021). Poly-ADP-ribosylation Drives Loss of Protein Homeostasis in ATM and Mre11 Deficiency. Mol. Cel 81 (7), 1515-1533. e5. doi:10.1016/j.molcel.2021.01.019

Lee, J. H., Mand, M. R., Kao, C. H., Zhou, Y., Ryu, S. W., Richards, A. L., et al. (2018). ATM Directs DNA Damage Responses and Proteostasis via Genetically Separable Pathways. Sci. Signal. 11 (512), eaan5598. doi:10.1126/scisignal.aan5598

Lezaja, A., and Altmeyer, M. (2018). Inherited DNA Lesions Determine G1 Duration in the Next Cell Cycle. Cell Cycle 17 (1), 24-32. doi:10.1080/ 15384101.2017.1383578

Lin, Y., Protter, D. S. W., Rosen, M. K., and Parker, R. (2015). Formation and Maturation of Phase-Separated Liquid Droplets by RNA-Binding Proteins. Mol. Cel 60 (2), 208-219. doi:10.1016/j.molcel.2015.08.018

Lisby, M., Mortensen, U. H., and Rothstein, R. (2003). Colocalization of Multiple DNA Double-Strand Breaks at a Single Rad52 Repair centre. Nat. Cel Biol 5 (6), 572-577. doi:10.1038/ncb997

Loomis, E. W., Sanz, L. A., Chédin, F., and Hagerman, P. J. (2014). Transcriptionassociated R-Loop Formation across the Human FMR1 CGG-Repeat Region. Plos Genet. 10 (4), e1004294. doi:10.1371/journal.pgen.1004294

Lord, C. J., and Ashworth, A. (2017). PARP Inhibitors: Synthetic Lethality in the Clinic. Science 355 (6330), 1152-1158. doi:10.1126/science.aam7344

Lottersberger, F., Karssemeijer, R. A., Dimitrova, N., and de Lange, T. (2015). 53BP1 and the LINC Complex Promote Microtubule-dependent DSB Mobility and DNA Repair. Cell 163 (4), 880-893. doi:10.1016/j.cell.2015.09.057

Lukas, C., Savic, V., Bekker-Jensen, S., Doil, C., Neumann, B., Sølvhøj Pedersen, R., et al. (2011). 53BP1 Nuclear Bodies Form Around DNA Lesions Generated by Mitotic Transmission of Chromosomes under Replication Stress. Nat. Cel Biol 13 (3), 243-253. doi:10.1038/ncb2201

Madabhushi, R., Gao, F., Pfenning, A. R., Pan, L., Yamakawa, S., Seo, J., et al. (2015). Activity-Induced DNA Breaks Govern the Expression of Neuronal Early-Response Genes. Cell 161 (7), 1592-1605. doi:10.1016/j.cell.2015.05.032

Marchal, C., Sima, J., and Gilbert, D. M. (2019). Control of DNA Replication Timing in the 3D Genome. Nat. Rev. Mol. Cel Biol 20 (12), 721-737. doi:10.1038/s41580-019-0162-y

McSwiggen, D. T., Mir, M., Darzacq, X., and Tjian, R. (2019). Evaluating Phase Separation in Live Cells: Diagnosis, Caveats, and Functional Consequences. Genes Dev. 33 (23-24), 1619-1634. doi:10.1101/gad.331520.119
Meisenberg, C., Pinder, S. I., Hopkins, S. R., Wooller, S. K., Benstead-Hume, G., Pearl, F. M. G., et al. (2019). Repression of Transcription at DNA Breaks Requires Cohesin throughout Interphase and Prevents Genome Instability. Mol. Cell 73 (2), 212-223. e7. doi:10.1016/j.molcel.2018.11.001

Miné-Hattab, J., Heltberg, M., Villemeur, M., Guedj, C., Mora, T., Walczak, A. M., et al. (2021). Single Molecule Microscopy Reveals Key Physical Features of Repair Foci in Living Cells. Elife 10, e60577. doi:10.7554/eLife.60577

Miné-Hattab, J., and Chiolo, I. (2020). Complex Chromatin Motions for DNA Repair. Front. Genet. 11, 800. doi:10.3389/fgene.2020.00800

Miné-Hattab, J., and Rothstein, R. (2012). Increased Chromosome Mobility Facilitates Homology Search during Recombination. Nat. Cel Biol 14 (5), 510-517. doi:10.1038/ncb2472

Miné-Hattab, J., and Taddei, A. (2019). Physical Principles and Functional Consequences of Nuclear Compartmentalization in Budding Yeast. Curr. Opin. Cel Biol. 58, 105-113. doi:10.1016/j.ceb.2019.02.005

Misteli, T. (2020). The Self-Organizing Genome: Principles of Genome Architecture and Function. Cell 183 (1), 28-45. doi:10.1016/j.cell.2020.09.014

Mizuno, K. I., Miyabe, I., Schalbetter, S. A., Carr, A. M., and Murray, J. M. (2013). Recombination-restarted Replication Makes Inverted Chromosome Fusions at Inverted Repeats. Nature 493 (7431), 246-249. doi:10.1038/nature11676

Monahan, Z., Ryan, V. H., Janke, A. M., Burke, K. A., Rhoads, S. N., Zerze, G. H., et al. (2017). Phosphorylation of the FUS Low-complexity Domain Disrupts Phase Separation, Aggregation, and Toxicity. EMBO J. 36 (20), 2951-2967. doi:10.15252/embj.201696394

Moreira, M.-C., Klur, S., Watanabe, M., Németh, A. H., Ber, I. L., Moniz, J.-C., et al. (2004). Senataxin, the Ortholog of a Yeast RNA Helicase, Is Mutant in AtaxiaOcular Apraxia 2. Nat. Genet. 36 (3), 225-227. doi:10.1038/ng1303

Murray, D. T., Kato, M., Lin, Y., Thurber, K. R., Hung, I., McKnight, S. L., et al. (2017). Structure of FUS Protein Fibrils and its Relevance to Self-Assembly and Phase Separation of Low-Complexity Domains. Cell 171 (3), 615-627. e16. doi:10.1016/j.cell.2017.08.048

Nagai, S., Dubrana, K., Tsai-Pflugfelder, M., Davidson, M. B., Roberts, T. M., Brown, G. W., et al. (2008). Functional Targeting of DNA Damage to a Nuclear Pore-Associated SUMO-dependent Ubiquitin Ligase. Science 322 (5901), 597-602. doi:10.1126/science.1162790

Natale, F., Rapp, A., Yu, W., Maiser, A., Harz, H., Scholl, A., et al. (2017). Identification of the Elementary Structural Units of the DNA Damage Response. Nat. Commun. 8, 15760. doi:10.1038/ncomms15760

Ochs, F., Karemore, G., Miron, E., Brown, J., Sedlackova, H., Rask, M.-B., et al. (2019). Stabilization of Chromatin Topology Safeguards Genome Integrity. Nature 574 (7779), 571-574. doi:10.1038/s41586-019-1659-4

Ohle, C., Tesorero, R., Schermann, G., Dobrev, N., Sinning, I., and Fischer, T. (2016). Transient RNA-DNA Hybrids Are Required for Efficient DoubleStrand Break Repair. Cell 167 (4), 1001-1013. e7. doi:10.1016/ j.cell.2016.10.001

Oshidari, R., Huang, R., Medghalchi, M., Tse, E. Y. W., Ashgriz, N., Lee, H. O., et al. (2020). DNA Repair by Rad52 Liquid Droplets. Nat. Commun. 11 (1), 695. doi:10.1038/s41467-020-14546-z

Oshidari, R., Strecker, J., Chung, D. K. C., Abraham, K. J., Chan, J. N. Y., Damaren, C. J., et al. (2018). Nuclear Microtubule Filaments Mediate Non-linear Directional Motion of Chromatin and Promote DNA Repair. Nat. Commun. 9 (1), 2567. doi:10.1038/s41467-018-05009-7

Parisis, N., Krasinska, L., Harker, B., Urbach, S., Rossignol, M., Camasses, A., et al. (2017). Initiation of DNA Replication Requires Actin Dynamics and Formin Activity. EMBO J. 36 (21), 3212-3231. doi:10.15252/embj.201796585

Patel, A., Lee, H. O., Jawerth, L., Maharana, S., Jahnel, M., Hein, M. Y., et al. (2015). A Liquid-To-Solid Phase Transition of the ALS Protein FUS Accelerated by Disease Mutation. Cell 162 (5), 1066-1077. doi:10.1016/j.cell.2015.07.047

Perego, M. G. L., Taiana, M., Bresolin, N., Comi, G. P., and Corti, S. (2019). R-loops in Motor Neuron Diseases. Mol. Neurobiol. 56 (4), 2579-2589. doi:10.1007/ s12035-018-1246-y

Pessina, F., Giavazzi, F., Yin, Y., Gioia, U., Vitelli, V., Galbiati, A., et al. (2019). Functional Transcription Promoters at DNA Double-Strand Breaks Mediate RNA-Driven Phase Separation of Damage-Response Factors. Nat. Cel Biol 21 (10), 1286-1299. doi:10.1038/s41556-019-0392-4

Polo, S. E., and Jackson, S. P. (2011). Dynamics of DNA Damage Response Proteins at DNA Breaks: a Focus on Protein Modifications. Genes Develop. 25 (5), 409-433. doi:10.1101/gad.2021311 
Pope, B. D., Ryba, T., Dileep, V., Yue, F., Wu, W., Denas, O., et al. (2014). Topologically Associating Domains Are Stable Units of Replication-Timing Regulation. Nature 515 (7527), 402-405. doi:10.1038/nature13986

Potts, P. R., Porteus, M. H., and Yu, H. (2006). Human SMC5/6 Complex Promotes Sister Chromatid Homologous Recombination by Recruiting the SMC1/3 Cohesin Complex to Double-Strand Breaks. EMBO J. 25 (14), 3377-3388. doi:10.1038/sj.emboj.7601218

Price, B. D., and D'Andrea, A. D. (2013). Chromatin Remodeling at DNA DoubleStrand Breaks. Cell 152 (6), 1344-1354. doi:10.1016/j.cell.2013.02.011

Rao, S. S. P., Huang, S.-C., Glenn St Hilaire, B., Engreitz, J. M., Perez, E. M., KiefferKwon, K.-R., et al. (2017). Cohesin Loss Eliminates All Loop Domains. Cell 171 (2), 305-320. e24. doi:10.1016/j.cell.2017.09.026

Rao, S. S. P., Huntley, M. H., Durand, N. C., Stamenova, E. K., Bochkov, I. D., Robinson, J. T., et al. (2014). A 3D Map of the Human Genome at Kilobase Resolution Reveals Principles of Chromatin Looping. Cell 159 (7), 1665-1680. doi:10.1016/j.cell.2014.11.021

Ray Chaudhuri, A., and Nussenzweig, A. (2017). The Multifaceted Roles of PARP1 in DNA Repair and Chromatin Remodelling. Nat. Rev. Mol. Cel Biol 18 (10), 610-621. doi:10.1038/nrm.2017.53

Regal, J. A., Festerling, T. A., Buis, J. M., and Ferguson, D. O. (2013). Diseaseassociated MRE11 Mutants Impact ATM/ATR DNA Damage Signaling by Distinct Mechanisms. Hum. Mol. Genet. 22 (25), 5146-5159. doi:10.1093/hmg/ ddt368

Reynolds, J. J., Bicknell, L. S., Carroll, P., Higgs, M. R., Shaheen, R., Murray, J. E., et al. (2017). Mutations in DONSON Disrupt Replication fork Stability and Cause Microcephalic Dwarfism. Nat. Genet. 49 (4), 537-549. doi:10.1038/ ng. 3790

Rogakou, E. P., Boon, C., Redon, C., and Bonner, W. M. (1999). Megabase Chromatin Domains Involved in DNA Double-Strand Breaks In Vivo. J. Cel Biol 146 (5), 905-916. doi:10.1083/jcb.146.5.905

Rogakou, E. P., Pilch, D. R., Orr, A. H., Ivanova, V. S., and Bonner, W. M. (1998). DNA Double-Stranded Breaks Induce Histone H2AX Phosphorylation on Serine 139. J. Biol. Chem. 273 (10), 5858-5868. doi:10.1074/jbc.273.10.5858

Rondinelli, B., Gogola, E., Yücel, H., Duarte, A. A., van de Ven, M., van der Sluijs, R., et al. (2017). EZH2 Promotes Degradation of Stalled Replication forks by Recruiting MUS81 through Histone H3 Trimethylation. Nat. Cel Biol 19 (11), 1371-1378. doi:10.1038/ncb3626

Roukos, V., and Misteli, T. (2014). The Biogenesis of Chromosome Translocations. Nat. Cel Biol 16 (4), 293-300. doi:10.1038/ncb2941

Roukos, V., Voss, T. C., Schmidt, C. K., Lee, S., Wangsa, D., and Misteli, T. (2013). Spatial Dynamics of Chromosome Translocations in Living Cells. Science 341 (6146), 660-664. doi:10.1126/science.1237150

Rowley, M. J., and Corces, V. G. (2018). Organizational Principles of 3D Genome Architecture. Nat. Rev. Genet. 19 (12), 789-800. doi:10.1038/s41576-0180060-8

Ruiz, P. D., Hamilton, G. A., Park, J. W., and Gamble, M. J. (2019). MacroH2A1 Regulation of Poly(ADP-Ribose) Synthesis and Stability Prevents Necrosis and Promotes DNA Repair. Mol. Cel Biol 40 (1), e00230-19. doi:10.1128/ MCB.00230-19

Ryu, T., Spatola, B., Delabaere, L., Bowlin, K., Hopp, H., Kunitake, R., et al. (2015). Heterochromatic Breaks Move to the Nuclear Periphery to Continue Recombinational Repair. Nat. Cel Biol 17 (11), 1401-1411. doi:10.1038/ ncb3258

Sanders, J. T., Freeman, T. F., Xu, Y., Golloshi, R., Stallard, M. A., Hill, A. M., et al. (2020). Radiation-induced DNA Damage and Repair Effects on 3D Genome Organization. Nat. Commun. 11 (1), 6178. doi:10.1038/s41467-020-20047-w

Sanulli, S., Trnka, M. J., Dharmarajan, V., Tibble, R. W., Pascal, B. D., Burlingame, A. L., et al. (2019). HP1 Reshapes Nucleosome Core to Promote Phase Separation of Heterochromatin. Nature 575 (7782), 390-394. doi:10.1038/ s41586-019-1669-2

Sarni, D., Sasaki, T., Irony Tur-Sinai, M., Miron, K., Rivera-Mulia, J. C., Magnuson, B., et al. (2020). 3D Genome Organization Contributes to Genome Instability at Fragile Sites. Nat. Commun. 11 (1), 3613. doi:10.1038/s41467-020-17448-2

Schep, R., Brinkman, E. K., Leemans, C., Vergara, X., van der Weide, R. H., Morris, B., et al. (2021). Impact of Chromatin Context on Cas9-Induced DNA DoubleStrand Break Repair Pathway Balance. Mol. Cel 81 (10), 2216-2230. el0. doi:10.1016/j.molcel.2021.03.032
Schrank, B., and Gautier, J. (2019). Assembling Nuclear Domains: Lessons from DNA Repair. J. Cel Biol 218 (8), 2444-2455. doi:10.1083/jcb.201904202

Schrank, B. R., Aparicio, T., Li, Y., Chang, W., Chait, B. T., Gundersen, G. G., et al. (2018). Nuclear ARP2/3 Drives DNA Break Clustering for Homology-Directed Repair. Nature 559 (7712), 61-66. doi:10.1038/s41586-018-0237-5

Scully, R., Panday, A., Elango, R., and Willis, N. A. (2019). DNA Double-Strand Break Repair-Pathway Choice in Somatic Mammalian Cells. Nat. Rev. Mol. Cel Biol 20 (11), 698-714. doi:10.1038/s41580-019-0152-0

Sebastian, R., and Oberdoerffer, P. (2017). Transcription-associated Events Affecting Genomic Integrity. Philos. Trans. R. Soc. Lond. B Biol. Sci. 372 (1731), 20160288. doi:10.1098/rstb.2016.0288

Sebastian, R., Hosogane, E. K., Sun, E. G., Tran, A. D., Reinhold, W. C., Burkett, S., et al. (2020). Epigenetic Regulation of DNA Repair Pathway Choice by MacroH2A1 Splice Variants Ensures Genome Stability. Mol. Cel 79 (5), 836-845. e7. doi:10.1016/j.molcel.2020.06.028

Shiloh, Y. (2020). The Cerebellar Degeneration in Ataxia-Telangiectasia: A Case for Genome Instability. DNA Repair 95, 102950. doi:10.1016/j.dnarep.2020.102950

Shin, Y., Berry, J., Pannucci, N., Haataja, M. P., Toettcher, J. E., and Brangwynne, C. P. (2017). Spatiotemporal Control of Intracellular Phase Transitions Using Light-Activated optoDroplets. Cell 168 (1-2), 159-e14. e14. doi:10.1016/ j.cell.2016.11.054

Singatulina, A. S., Hamon, L., Sukhanova, M. V., Desforges, B., Joshi, V., Bouhss, A., et al. (2019). PARP-1 Activation Directs FUS to DNA Damage Sites to Form PARG-Reversible Compartments Enriched in Damaged DNA. Cel Rep. 27 (6), 1809-1821. e5. doi:10.1016/j.celrep.2019.04.031

Singh, G., Pratt, G., Yeo, G. W., and Moore, M. J. (2015). The Clothes Make the mRNA: Past and Present Trends in mRNP Fashion. Annu. Rev. Biochem. 84, 325-354. doi:10.1146/annurev-biochem-080111-092106

Smeenk, G., and van Attikum, H. (2013). The Chromatin Response to DNA Breaks: Leaving a Mark on Genome Integrity. Annu. Rev. Biochem. 82, 55-80. doi:10.1146/annurev-biochem-061809-174504

Smith, M. J., and Rothstein, R. (2017). Poetry in Motion: Increased Chromosomal Mobility after DNA Damage. DNA Repair 56, 102-108. doi:10.1016/ j.dnarep.2017.06.012

Soutoglou, E., Dorn, J. F., Sengupta, K., Jasin, M., Nussenzweig, A., Ried, T., et al. (2007). Positional Stability of Single Double-Strand Breaks in Mammalian Cells. Nat. Cel Biol 9 (6), 675-682. doi:10.1038/ncb1591

Spies, J., Lukas, C., Somyajit, K., Rask, M.-B., Lukas, J., and Neelsen, K. J. (2019). 53BP1 Nuclear Bodies Enforce Replication Timing at Under-replicated DNA to Limit Heritable DNA Damage. Nat. Cel Biol 21 (4), 487-497. doi:10.1038/ s41556-019-0293-6

Strom, A. R., Emelyanov, A. V., Mir, M., Fyodorov, D. V., Darzacq, X., and Karpen, G. H. (2017). Phase Separation Drives Heterochromatin Domain Formation. Nature 547 (7662), 241-245. doi:10.1038/nature22989

Ström, L., Lindroos, H. B., Shirahige, K., and Sjögren, C. (2004). Postreplicative Recruitment of Cohesin to Double-Strand Breaks Is Required for DNA Repair. Mol. Cel 16 (6), 1003-1015. doi:10.1016/j.molcel.2004.11.026

Symington, L. S., and Gautier, J. (2011). Double-strand Break End Resection and Repair Pathway Choice. Annu. Rev. Genet. 45, 247-271. doi:10.1146/annurevgenet-110410-132435

Szabo, Q., Bantignies, F., and Cavalli, G. (2019). Principles of Genome Folding into Topologically Associating Domains. Sci. Adv. 5 (4), eaaw1668. doi:10.1126/ sciadv.aaw1668

Taslimi, A., Vrana, J. D., Chen, D., Borinskaya, S., Mayer, B. J., Kennedy, M. J., et al. (2014). An Optimized Optogenetic Clustering Tool for Probing Protein Interaction and Function. Nat. Commun. 5, 4925. doi:10.1038/ncomms5925

Taylor, A. M., Groom, A., and Byrd, P. J. (2004). Ataxia-telangiectasia-like Disorder (ATLD)-its Clinical Presentation and Molecular Basis. DNA Repair (Amst) 3 (8-9), 1219-1225. doi:10.1016/j.dnarep.2004.04.009

Tsouroula, K., Furst, A., Rogier, M., Heyer, V., Maglott-Roth, A., Ferrand, A., et al. (2016). Temporal and Spatial Uncoupling of DNA Double Strand Break Repair Pathways within Mammalian Heterochromatin. Mol. Cel 63 (2), 293-305. doi:10.1016/j.molcel.2016.06.002

Ünal, E., Arbel-Eden, A., Sattler, U., Shroff, R., Lichten, M., Haber, J. E., et al. (2004). DNA Damage Response Pathway Uses Histone Modification to Assemble a Double-Strand Break-specific Cohesin Domain. Mol. Cel 16 (6), 991-1002. doi:10.1016/j.molcel.2004.11.027 
Uusküla-Reimand, L., Hou, H., Samavarchi-Tehrani, P., Rudan, M. V., Liang, M., Medina-Rivera, A., et al. (2016). Topoisomerase II Beta Interacts with Cohesin and CTCF at Topological Domain Borders. Genome Biol. 17 (1), 182. doi:10.1186/s13059-016-1043-8

Wahba, L., Gore, S. K., and Koshland, D. (2013). The Homologous Recombination Machinery Modulates the Formation of RNA-DNA Hybrids and Associated Chromosome Instability. Elife 2, e00505. doi:10.7554/eLife.00505

Wang, L., Gao, Y., Zheng, X., Liu, C., Dong, S., Li, R., et al. (2019). Histone Modifications Regulate Chromatin Compartmentalization by Contributing to a Phase Separation Mechanism. Mol. Cel 76 (4), 646-659. doi:10.1016/ j.molcel.2019.08.019

Whalen, J. M., Dhingra, N., Wei, L., Zhao, X., and Freudenreich, C. H. (2020). Relocation of Collapsed Forks to the Nuclear Pore Complex Depends on Sumoylation of DNA Repair Proteins and Permits Rad51 Association. Cel Rep. 31 (6), 107635. doi:10.1016/j.celrep.2020.107635

Witcher, M., and Emerson, B. M. (2009). Epigenetic Silencing of the p16INK4a Tumor Suppressor Is Associated with Loss of CTCF Binding and a Chromatin Boundary. Mol. Cel 34 (3), 271-284. doi:10.1016/ j.molcel.2009.04.001

Wright, R. L., and Vaughan, A. T. M. (2014). A Systematic Description of MLL Fusion Gene Formation. Crit. Rev. Oncology/Hematology 91 (3), 283-291. doi:10.1016/j.critrevonc.2014.03.004

Yatskevich, S., Rhodes, J., and Nasmyth, K. (2019). Organization of Chromosomal DNA by SMC Complexes. Annu. Rev. Genet. 53, 445-482. doi:10.1146/ annurev-genet-112618-043633

Zhang, J., Bellani, M. A., James, R. C., Pokharel, D., Zhang, Y., Reynolds, J. J., et al. (2020). DONSON and FANCM Associate with Different Replisomes Distinguished by Replication Timing and Chromatin Domain. Nat. Commun. 11 (1), 3951. doi:10.1038/s41467-020-17449-1
Zhang, X., Zhang, Y., Ba, Z., Kyritsis, N., Casellas, R., and Alt, F. W. (2019). Fundamental Roles of Chromatin Loop Extrusion in Antibody Class Switching. Nature 575 (7782), 385-389. doi:10.1038/s41586-019-1723-0

Zhang, Y., Zhang, X., Ba, Z., Liang, Z., Dring, E. W., Hu, H., et al. (2019). The Fundamental Role of Chromatin Loop Extrusion in Physiological V(D)J Recombination. Nature 573 (7775), 600-604. doi:10.1038/s41586-019-1547-y

Zhen, Y., Zhang, Y., and Yu, Y. (2017). A Cell-line-specific Atlas of PARPMediated Protein Asp/Glu-ADP-Ribosylation in Breast Cancer. Cel Rep. 21 (8), 2326-2337. doi:10.1016/j.celrep.2017.10.106

Zong, D., Oberdoerffer, P., Batista, P. J., and Nussenzweig, A. (2020). RNA: a Double-Edged Sword in Genome Maintenance. Nat. Rev. Genet. 21 (11), 651-670. doi:10.1038/s41576-020-0263-7

Conflict of Interest: The authors declare that the research was conducted in the absence of any commercial or financial relationships that could be construed as a potential conflict of interest.

Publisher's Note: All claims expressed in this article are solely those of the authors and do not necessarily represent those of their affiliated organizations, or those of the publisher, the editors and the reviewers. Any product that may be evaluated in this article, or claim that may be made by its manufacturer, is not guaranteed or endorsed by the publisher.

Copyright (c) 2021 Sebastian, Aladjem and Oberdoerffer. This is an open-access article distributed under the terms of the Creative Commons Attribution License (CC $B Y)$. The use, distribution or reproduction in other forums is permitted, provided the original author(s) and the copyright owner(s) are credited and that the original publication in this journal is cited, in accordance with accepted academic practice. No use, distribution or reproduction is permitted which does not comply with these terms. 